NASA/CR-2003-212387

\title{
On a Non-Reflecting Boundary Condition for Hyperbolic Conservation Laws
}

Ching Y. Loh

Taitech, Inc., Beaver Creek, Ohio 
Since its founding, NASA has been dedicated to the advancement of aeronautics and space science. The NASA Scientific and Technical Information (STI) Program Office plays a key part in helping NASA maintain this important role.

The NASA STI Program Office is operated by Langley Research Center, the Lead Center for NASA's scientific and technical information. The NASA STI Program Office provides access to the NASA STI Database, the largest collection of aeronautical and space science STI in the world. The Program Office is also NASA's institutional mechanism for disseminating the results of its research and development activities. These results are published by NASA in the NASA STI Report Series, which includes the following report types:

- $\quad$ TECHNICAL PUBLICATION. Reports of completed research or a major significant phase of research that present the results of NASA programs and include extensive data or theoretical analysis. Includes compilations of significant scientific and technical data and information deemed to be of continuing reference value. NASA's counterpart of peerreviewed formal professional papers but has less stringent limitations on manuscript length and extent of graphic presentations.

- TECHNICAL MEMORANDUM. Scientific and technical findings that are preliminary or of specialized interest, e.g., quick release reports, working papers, and bibliographies that contain minimal annotation. Does not contain extensive analysis.

- CONTRACTOR REPORT. Scientific and technical findings by NASA-sponsored contractors and grantees.
- CONFERENCE PUBLICATION. Collected papers from scientific and technical conferences, symposia, seminars, or other meetings sponsored or cosponsored by NASA.

- SPECIAL PUBLICATION. Scientific, technical, or historical information from NASA programs, projects, and missions, often concerned with subjects having substantial public interest.

- TECHNICAL TRANSLATION. Englishlanguage translations of foreign scientific and technical material pertinent to NASA's mission.

Specialized services that complement the STI Program Office's diverse offerings include creating custom thesauri, building customized databases, organizing and publishing research results ... even providing videos.

For more information about the NASA STI Program Office, see the following:

- Access the NASA STI Program Home Page at http://www.sti.nasa.gov

- E-mail your question via the Internet to help@sti.nasa.gov

- Fax your question to the NASA Access Help Desk at 301-621-0134

- Telephone the NASA Access Help Desk at 301-621-0390

- Write to:

NASA Access Help Desk

NASA Center for AeroSpace Information 7121 Standard Drive

Hanover, MD 21076 
NASA/CR-2003-212387

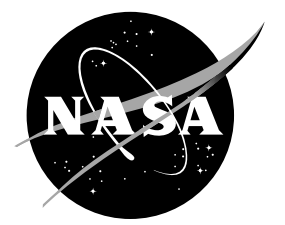

\section{On a Non-Reflecting Boundary Condition for Hyperbolic Conservation Laws}

Ching Y. Loh

Taitech, Inc., Beaver Creek, Ohio

Prepared for the

16th Computational Fluid Dynamics Conference and the 33rd Fluid Dynamics Conference and Exhibit

sponsored by the American Institute of Aeronautics and Astronautics

Orlando, Florida, June 23-26, 2003

Prepared under Contract NAS3-03072

National Aeronautics and

Space Administration

Glenn Research Center 


\section{Acknowledgments}

This work received support from the Supersonic Propulsion Technology Project Office of NASA Glenn Research Center. The author wishes to thank Dr. R. Hixon, Dr. R. Blech, Dr. P.C.E. Jorgenson, Dr. A. Himansu, and Dr. X.Y. Wang for fruitful discussions on the issues in the present paper.

This report contains preliminary findings, subject to revision as analysis proceeds.

The Propulsion and Power Program at NASA Glenn Research Center sponsored this work.

Available from

NASA Center for Aerospace Information 7121 Standard Drive

Hanover, MD 21076
National Technical Information Service 5285 Port Royal Road Springfield, VA 22100 


\title{
ON A NON-REFLECTING BOUNDARY CONDITION FOR HYPERBOLIC CONSERVATION LAWS
}

\author{
Ching Y. Loh \\ Taitech, Inc. \\ Beaver Creek, Ohio 45430 \\ Email: fsloh@turbot.grc.nasa.gov
}

\begin{abstract}
A non-reflecting boundary condition (NRBC) for practical computations in fluid dynamics and aeroacoustics is presented. The technique is based on the first principle of non-reflecting, plane wave propagation [1] and the hyperbolicity of the Euler equation system. The NRBC is simple and effective, provided the numerical scheme maintains locally a $C^{1}$ continuous solution at the boundary. Several numerical examples in 1D, 2D and 3D space are illustrated to demonstrate its robustness in practical computations.
\end{abstract}

\section{Introduction}

It is well-known that non-reflecting boundary conditions (NRBCs) play an important role in fluid flow and aeroacoustics computations. The need for artificial boundary conditions arises when the domain of the problem is unbounded and extends to infinity. In order to treat the problem numerically, a domain of finite size is required and artificial boundaries are imposed. At these artificial boundaries, NRBCs are sought for to minimize their influences on the flow. A spurious reflection resulting from an inappropriate numerical boundary condition will contaminate the flow field and may entirely spoil the flow computation. Research on NRBC is a challenging topic in engineering and applied mathematics. For decades, a vast number of papers on NRBC have been published, e.g. see [3-6], the review paper by Givoli [2], and the references cited there.

In one-dimensional flow, at an artificial boundary, Enquist and Majda [3], and Hedstrom [7] proved that a boundary condition is non-reflecting is equivalent to saying that the characteristic variables corresponding to the incoming characteristic curve remain constant across the artificial boundary (see also Hirsch [8], p. 370). For multi-dimensional flow, this 1-D technique is combined with dimension splitting and applied in the practical NRBC treatment. Such a combined treatment has been the topic of many papers on the characteristics based NRBCs (see e.g. [4] and references in [2]). 
Other alternative treatments for NRBC found in the literature include (see [2-6]):

(i) in the far-field, using a predictable asymptotic analytical solution at the boundary ( the radiation boundary condition),

(ii) diminishing the strength of the waves/disturbances before they reach the artificial boundary, and thus minimizing the reflecting effect. Usually, increased numerical damping is applied to a zone between the core domain and the artificial boundary (the buffer zone or sponge zone) to do the job.

(iii) In the recently developed PML (perfectly matched layer) method, a specially designed equation system is imposed in the matching layer (or sponge zone) to guarantee the exponential decaying of the disturbances in the layer $[5,6]$.

In the present paper, a different but simple criterion is introduced to treat the NRBCs of the time-dependent hyperbolic conservation laws of gas dynamics. The criterion is based on the first principle of non-reflecting and plane wave propagation [1] rather than the characteristics theory.

As it turns out that the NRBCs used in the recent CE/SE finite volume schemes for flow and aeroacoustics computations (e.g. $[16,17])$ can be directly derived from this criterion, the present paper also serves to explain why these simple NRBCs works well with the CE/SE schemes.

The paper is arranged as follows: In Section 2, based on the first principle of non-reflecting, the propagation of plane wave and the hyperbolicity of the Euler equation system the continuity criterion of NRBC is introduced and proved. The numerical treatment of the continuity criterion is presented in $\S 3$, an extrapolation-like NRBC (Type I) based on this criterion and the numerical procedure are described. In $\S 4$ the relation between the NRBC and the flux balance across the boundary surface is established, which leads to another type of NRBC (Type II). Discussions on the NRBCs are given in $\S 5$. In Section 6, several numerical examples for outflow NRBC in one and multi-dimensional space are presented. Numerical examples with Type II NRBC at the inflow and other artificial boundaries are demonstrated in Section 7. Application of buffer/sponge zones is illustrated in Section 8. At last, the paper is concluded with remarks in Section 9.

As the time-dependent hyperbolic conservation laws of gas dynamics ( in dimensionless form) is always incorporated in the NRBCs in the present paper, they are briefly described here for later use:

$$
\boldsymbol{U}_{t}+\boldsymbol{F}_{x}+\boldsymbol{G}_{y}+\boldsymbol{H}_{z}=\mathbf{Q}
$$

where $x, y, z$ and $t$ are the streamwise and transversal coordinates and time, respectively. The conservative flow 
variable vector $\boldsymbol{U}$ and the flux vectors in the streamwise and radial directions, $\boldsymbol{F}, \boldsymbol{G}$, and $\boldsymbol{H}$, are given by:

$$
\begin{gathered}
\boldsymbol{U}=\left(\begin{array}{l}
U_{1} \\
U_{2} \\
U_{3} \\
U_{4} \\
U_{5}
\end{array}\right)=\left(\begin{array}{c}
\rho \\
\rho u \\
\rho v \\
\rho w \\
\rho e
\end{array}\right), \boldsymbol{F}=\left(\begin{array}{c}
F_{1} \\
F_{2} \\
F_{3} \\
F_{4} \\
F_{5}
\end{array}\right)=\left(\begin{array}{c}
\rho u \\
\rho u^{2}+p \\
\rho u v \\
\rho u w \\
\rho u H
\end{array}\right), \\
\boldsymbol{G}=\left(\begin{array}{l}
G_{1} \\
G_{2} \\
G_{3} \\
G_{4} \\
G_{5}
\end{array}\right)=\left(\begin{array}{c}
\rho v \\
\rho u v \\
\rho v^{2}+p \\
\rho v w \\
\rho v H
\end{array}\right), \quad \boldsymbol{H}=\left(\begin{array}{l}
H_{1} \\
h_{2} \\
H_{3} \\
H_{4} \\
H_{5}
\end{array}\right)=\left(\begin{array}{c}
\rho w \\
\rho w u \\
\rho w v \\
\rho w^{2}+p \\
\rho w H
\end{array}\right) .
\end{gathered}
$$

where $u, v, w$ and $\rho, p$ are respectively the velocity components, density and pressure, $e=\frac{p}{\rho(\gamma-1)}+1 / 2\left(u^{2}+v^{2}+w^{2}\right)$, and the enthalpy $H=p / \rho+e$ with $\gamma=1$.4. The right hand side $\mathbf{Q}$ is the source term which may include the possible external forcing terms and/or viscous fluxes.

By considering $(x, y, z, t)$ as coordinates of a four-dimensional Euclidean space, $E_{4}$, and using Gauss's divergence theorem, it follows that Eq. (1) is equivalent to the following integral conservation laws:

$$
\oint_{S(V)} \mathbf{I}_{\mathbf{m}} \cdot \mathrm{d} \mathbf{s}=\int_{V} \mathbf{Q}_{\mathbf{m}} \mathrm{d} V, \quad m=1,2,3,4,5,
$$

where $S(V)$ denotes the surface around a volume $V$ in $E_{4}$ and $\mathbf{I}_{\mathbf{m}}=\left(F_{m}, G_{m}, H_{m}, U_{m}\right)$ stands for the flux vectors, $\mathbf{I}_{\mathbf{m}} \mathrm{d} \mathbf{s}=\mathbf{I}_{\mathbf{m}} \bullet \mathbf{n} d s, \mathbf{n}$ being the outgoing unit normal vector in $E_{4}$, is the flux at the infinitesimal surface element $d s$.

\section{The continuity criterion of NRBC}

In numerical schemes, particularly in finite volume numerical approaches with hyperbolic conservation laws, grid nodes are often cell centers and the boundary faces are often formed by the boundary cell surfaces. No node lies exactly on the boundary. As such, a continuity criterion of NRBC and the consequent NRBC treatments are introduced based on the first principle of non-reflecting, the first principle of plane wave propagation [1] and the hyperbolicity of the equation system. They are simple, robust and particularly appropriate for the cell center finite volume schemes. In the following, the continuity criterion is first introduced in an heuristic way and then proved via the first principle of non-reflecting and plane wave propagation [1]. Their limitations are also briefly discussed.

For simplicity, the flow is assumed to be continuous near the boundary, i.e., with no shock or contact discontinuity. Later, it will be shown, as a discontinuous wave (e.g. a shock) may be decomposed by Fourier integral or Fourier series into a series of plane waves of different wave numbers, the NRBC may still be valid for it. 


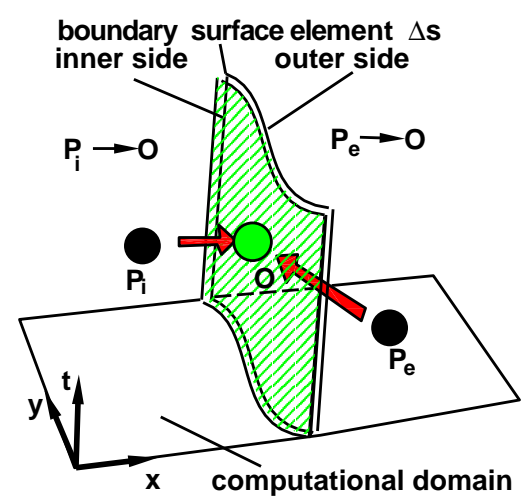

Figure 1: The NRBC criterion in $E_{3}$.

In the following context, $\mathbf{U}=\left(u_{1}, u_{2}, u_{3}, u_{4}, u_{5}\right)^{T}=(\rho, \rho u, \rho v, \rho w, \rho e)^{T}, \mathbf{V}=\left(v_{1}, v_{2}, v_{3}, v_{4}, v_{5}\right)^{T}=(\rho, u, v, w, p)^{T}$, and $\mathbf{W}=\left(w_{1}, w_{2}, w_{3}, w_{4}, w_{5}\right)^{T}$ are employed to represent respectively the conservative flow variables, the primitive flow variables and the characteristic variables.

\subsection{A heuristic approach}

We begin with the behavior of the characteristic variables $\mathbf{W}=\left(w_{1}, w_{2}, w_{3}, w_{4}, w_{5}\right)^{T}$ across an artificial boundary surface element $\Delta s$ as time elapses. $\Delta s$ also represents the interface between a boundary cell and its corresponding ghost cell. Note that any spatial boundary is a cylindrical hyper-surface in the space-time $E_{4}$ space (Fig. 1).

There are various techniques to treat the NRBC based on characteristics theory. For instance, a well-known 1D flow NRBC treatment by Enquist and Majda [3], and Hedstrom [7] is the requirement that the local perturbation (disturbance) along incoming characteristics be made vanish at the boundary (see [8], p.370). Let $W=\left(w_{1}, w_{2}, w_{3}\right)^{T}$ be the 1-D characteristic flow variables, the above requirement states that for those $k$ such that the corresponding characteristic enters the computational domain through the artificial boundary:

$$
\Delta w_{k}=0
$$

Let $P_{i}$ and $P_{e}$ be respectively an interior point and an exterior point of the computational domain in the $E_{4}$ space. Both $P_{i}$ and $P_{e}$ lie in the neighborhood of $O$, a point on $\Delta s$ (Fig. 1). Then,

$$
\Delta w_{k}=w_{k}\left(P_{i}\right)-w_{k}\left(P_{e}\right)=0, \text { for selected } k .
$$

When $P_{i}$ tends to $O$ from the interior of the domain and $P_{e}$ tends to $O$ from the exterior of the domain, the NRBC Eq. (3) becomes:

$$
\lim _{P_{i} \rightarrow O} w_{k}\left(P_{i}\right)=\lim _{P_{e} \rightarrow O} w_{k}\left(P_{e}\right)=w_{k}(O)
$$


for those selected $k \mathrm{~s}$. Therefore, the usual NRBC treatment is formally interpreted as a continuity problem of $w_{k}$ across the boundary surface.

Secondly, for the NRBC of multi-dimensional flow, we formally extend the continuity of $w_{k}$ across the boundary surface to all $k \mathrm{~s}$ rather than just a selected number of $k \mathrm{~s}$ :

$$
\lim _{P_{i} \rightarrow O} w_{k}\left(P_{i}\right)=\lim _{P_{e} \rightarrow O} w_{k}\left(P_{e}\right), \quad \text { for } \quad \text { all } \quad k .
$$

From the local equivalence of the characteristic variables $\mathbf{W}$ and $\mathbf{V}$ or $\mathbf{U}$ (see e.g. Hirsch[8], p.155-156), Eq. (5) is equivalent to the continuity of $\mathbf{V}$ or $\mathbf{U}$ across the artificial boundary surface:

$$
\lim _{P_{i} \rightarrow O} u_{k}\left(P_{i}\right)=\lim _{P_{e} \rightarrow O} u_{k}\left(P_{e}\right), \quad \text { for } \quad \text { all } \quad k
$$

or

$$
\lim _{P_{i} \rightarrow O} v_{k}\left(P_{i}\right)=\lim _{P_{e} \rightarrow O} v_{k}\left(P_{e}\right), \quad \text { for all } k
$$

It is advantageous to switch from the continuity relation (5) of $\mathbf{W}$ to that of $\mathbf{V}$ or $\mathbf{U}$, (6) or (7), since the latter can be treated in an easy way. At this stage, the continuity criterion of NRBC is heuristically inferred.

\section{2 the first principle of non-reflecting}

The above heuristic approach shows intuitively how the continuity criterion can be inferred from the characteristics NRBC. In the following, the continuity criterion will be proved, but first of all, a definition of 'non-reflecting' is needed:

Definition: An artificial boundary is said to be non-reflecting if the solutions of the hyperbolic p.d.e.s (partial differential equations) in the domain interior and domain exterior are mutually solution continuations of each other across the boundary surface.

In other words, both these solutions are but part of the solution in the entire domain. Hence, any point at the artificial boundary may be considered as an interior point in the entire domain and there is no reflection. This is the first principle of non-reflecting adopted in the present paper. If, in the particular case that simple wave-like solutions exist:

$$
\mathbf{V}=\tilde{\mathbf{V}} e^{i \theta}
$$

where $i=\sqrt{-1}$, then both the 'amplitude' $\tilde{\mathbf{V}}$ and the phase $\theta$ are required to be identical across the artificial boundary.

\subsection{Plane wave solutions of the Euler/N-S equations}

The plane wave solutions are based on the Cauchy's method of Fourier Integral (see Courant and Hilbert[1], pp.210211). Consider the Euler/N-S equations in non-conservation form:

$$
\frac{\partial \mathbf{V}}{\partial t}+\tilde{A} \frac{\partial \mathbf{V}}{\partial x}+\tilde{B} \frac{\partial \mathbf{V}}{\partial y}+\tilde{C} \frac{\partial \mathbf{V}}{\partial z}=\tilde{\mathbf{Q}}
$$


where $\tilde{A}, \tilde{B}$, and $\tilde{C}$ are the jacobian matrices and functions of $\mathbf{V}$. and the viscous terms may be included in the source term $\tilde{\mathbf{Q}}$.

In the neighborhood of a point $O\left(\mathbf{x}_{\mathbf{0}}, t_{0}\right)$ at the artificial boundary, (8) can be locally linearized by setting the jacobians $\tilde{A}, \tilde{B}, \tilde{C}$ to their values at $O$. Assume a plane wave with the form

$$
\mathbf{V}=\tilde{\mathbf{V}} e^{i \theta}
$$

and substitute in (8). Here $\theta=\theta(\mathbf{x}, t)=\mathbf{x} \bullet \mathbf{k}-\omega t$ is the phase of the plane wave, $i=\sqrt{-1} . \theta=$ const. stands for a characteristic surface or wave front [1]. (8) then becomes:

$$
\left(\frac{\partial \tilde{\mathbf{V}}}{\partial t}+\tilde{A} \frac{\partial \tilde{\mathbf{V}}}{\partial x}+\tilde{B} \frac{\partial \tilde{\mathbf{V}}}{\partial y}+\tilde{C} \frac{\partial \tilde{\mathbf{V}}}{\partial z}\right)+i(\tilde{K}-\omega I) \tilde{\mathbf{V}}=\tilde{\mathbf{Q}} e^{-i \theta}
$$

where $I$ is the $5 \times 5$ identity matrix. For any given wave number vector $\mathbf{k}=\left(k_{x}, k_{y}, k_{z}\right)$, from the hyperbolicity of ( 8$)$, real eigenvalues $\omega$ s exist such that $|\tilde{K}-\omega I|=0$ (dispersion relation), and so also 5 linearly independent eigenvectors $\mathbf{r}_{\mathbf{m}}, \mathrm{m}=1,2, \ldots, 5$ such that $\left(\tilde{K}-\omega_{m} I\right) \mathbf{r}_{m}=0$, where the matrix $\tilde{K}=k_{x} \tilde{A}+k_{y} \tilde{B}+k_{z} \tilde{C}$.

As the eigenvectors $\mathbf{r}_{m}$ are linearly independent, they may be used as the new basis for the 5 dimensional space and so $\tilde{\mathbf{V}}$ is converted into $\overline{\mathbf{V}}=M^{-1} \tilde{\mathbf{V}}$, where $M^{-1}$ is the jacobian matrix of the coordinate transformation. Let $\tilde{\mathbf{V}}=\bar{v}_{m} \mathbf{r}_{m}, \mathrm{~m}=1,2,3,4,5$ in turn, or, say, $\overline{\mathbf{V}}=\overline{\mathbf{V}}_{m}=\left(0, \bar{v}_{m}, 0,0,0\right)^{T}$ in turn, where $\bar{v}_{m}$ is the scalar function to be solved for, also let $\overline{\mathbf{Q}}=\bar{q}_{m} \mathbf{r}_{m}=\overline{\mathbf{Q}}_{m}$ in turn. Then for $\omega=\omega_{m}$, (8) becomes

$$
\frac{\partial \overline{\mathbf{V}}}{\partial t}+M^{-1} \tilde{A} M \frac{\partial \overline{\mathbf{V}}}{\partial x}+M^{-1} \tilde{B} M \frac{\partial \overline{\mathbf{V}}}{\partial y}+M^{-1} \tilde{C} M \frac{\partial \overline{\mathbf{V}}}{\partial z}=\overline{\mathbf{Q}} e^{-i\left(\mathbf{x} \bullet \mathbf{k}-\omega_{m} t\right)} .
$$

Note that for each $\overline{\mathbf{V}}_{m}$ or $\overline{\mathbf{Q}}_{m}$, only one component is non-zero $\left(\bar{v}_{m}\right.$ or $\left.\bar{q}_{m}\right)$. and the above equation is equivalent to a single scalar equation for $\bar{v}_{m}$. Totally, there are five such equations. Also notice that $\omega_{m}$ changes with $m$. Finally, the 'amplitude' $\tilde{\mathbf{V}}$ may be expressed as

$$
\tilde{\mathbf{V}}=M \sum_{m=1}^{5} \overline{\mathbf{V}}_{m} e^{i\left(\mathbf{x} \bullet \mathbf{k}-\omega_{m} t\right)} .
$$

The same result was also given in [8] (Vol.1, p.153). Thus, (8) supports plane wave solutions in the form of (9) Thus, (8) supports plane wave solutions in the form of (9).

For more general waves other than the simple plane waves, as (8) is locally linearized in the neighborhood of the boundary point $O\left(\mathbf{x}_{\mathbf{0}}, t_{0}\right)$, they may be decomposed by Fourier series or Fourier integral with respect to wave number $\mathrm{k}$ and replaced by the superposition of plane waves.

\subsection{Proof of the continuity criterion}

With the presence of the artificial boundary $s$ (hyper-surface), time-space $E_{4}$ is bisected into two portions, domain interior $D_{i}$ and domain exterior $D_{e}$ (Fig.2). Within each portion, the flow is governed by the same Eq. (1). From the 


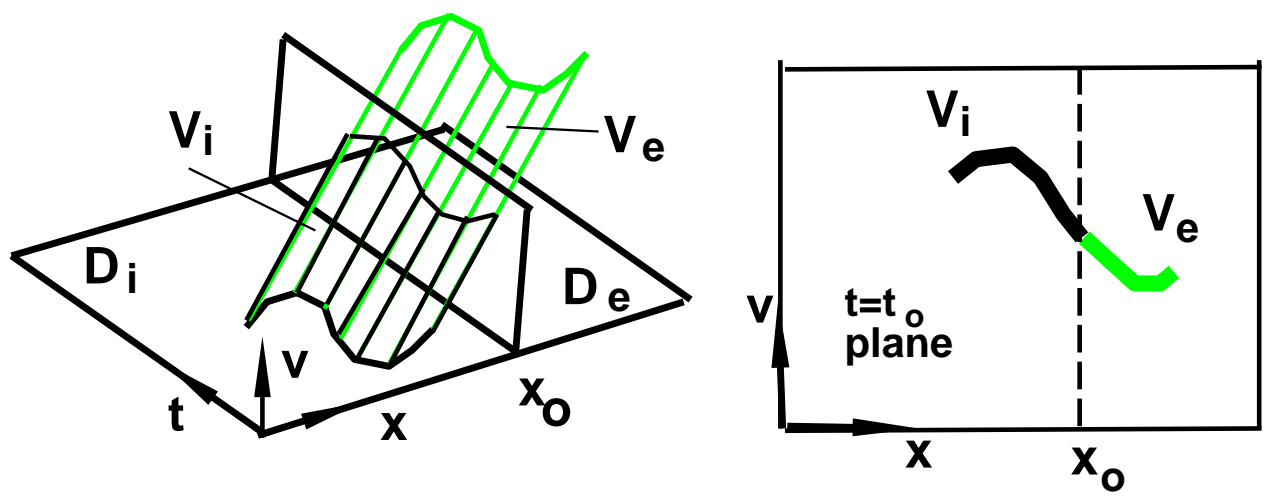

Figure 2: Sketch of the continuity criterion in 1-D flow, only a real component of $\mathbf{V}$ is shown.

first principle of non-reflecting and plane wave propagation, it can be shown that locally there is no reflection at the artificial boundary surface $s$ if the continuity criterion Eq. (7) (or Eq. (6), or Eq. (5) ) is satisfied.

Proof: Consider the non-conservation form (8):

$$
\frac{\partial \mathbf{V}}{\partial t}+\tilde{A} \frac{\partial \mathbf{V}}{\partial x}+\tilde{B} \frac{\partial \mathbf{V}}{\partial y}+\tilde{C} \frac{\partial \mathbf{V}}{\partial z}=\tilde{\mathbf{Q}}
$$

As a result of the continuity condition (7), an admissible given set of $\mathbf{V}$ at the boundary $s$ may be used as a common boundary condition to solve separately for $\mathbf{V}_{i}$ and $\mathbf{V}_{e}$ in their corresponding subdomains $D_{i}$ and $D_{e}$, which are separated by the artificial boundary $s$. (Note that generally, the admissible $\mathbf{V}$ given at $s$ should be identical to the solution of $\mathbf{V}$ over the entire domain, see Appendix ). Here, $\mathbf{V}_{i}$ and $\mathbf{V}_{e}$ are respectively the solutions of (8) in $D_{i}$ and $D_{e}$. Let $\mathbf{V}$ be the solution of (8) over the entire domain. Due to the uniqueness of solution for well-posed initialboundary value problems ( see Appendix ), $\mathbf{V}_{i}$ is identical to $\mathbf{V}$ in $D_{i}$ and $\mathbf{V}_{e}$ is identical to $\mathbf{V}$ in $D_{e}$. Therefore, in a neighborhood of $s, \mathbf{V}_{i}$ and $\mathbf{V}_{e}$ are mutually a continuation of each other across the boundary $s$ and hence there is no reflection according to the definition in $\S 2.2$.

To be more specific in terms of plane wave propagation, from $\S 2.3,(8)$ supports plane wave solutions. As a general wave solution can be locally written as a superposition of the plane wave solutions by Fourier integral, it suffices to consider only the behavior of a single plane wave solution in the form of (9) at the artificial boundary $s$.

Let $O\left(\mathbf{x}_{o}, t_{o}\right)$ be any point at the artificial boundary $s$, then (8) can be locally linearized in the neighborhood of $O$, i.e., $\tilde{A}, \tilde{B}$ and $\tilde{C}$ are frozen at $\mathbf{V}_{\mathbf{o}}$ as in $\S 2.3$. For any given wave number vector $\mathbf{k}$, from the continuity criterion (7), $\mathbf{V}_{o}=\mathbf{V}_{i}=\mathbf{V}_{e}$, all $\tilde{A}, \tilde{B}$ and $\tilde{C}$ remain the same across the boundary and so also the eigenvalues $\omega_{i}=\omega_{e}=\omega$. At $O$, the plane waves $\mathbf{V}_{i}$ and $\mathbf{V}_{e}$ share the same $\mathbf{k}, \mathbf{x}, \omega$ and $t$, and hence the same phase $\theta$. Again, from the continuity criterion (7), $\mathbf{V}_{i}=\mathbf{V}_{e}$, or

$$
\tilde{\mathbf{V}}_{i} e^{i(\mathbf{k} \bullet \mathbf{x}-\omega t)}=\tilde{\mathbf{V}}_{e} e^{i(\mathbf{k} \bullet \mathbf{x}-\omega t)},
$$

i.e., $\tilde{\mathbf{V}}_{i}=\tilde{\mathbf{V}}_{e}$. Thus, the plane waves $\mathbf{V}_{i}$ and $\mathbf{V}_{e}$ share the same amplitudes too. Therefore, $\mathbf{V}_{i}$ is completely identical 


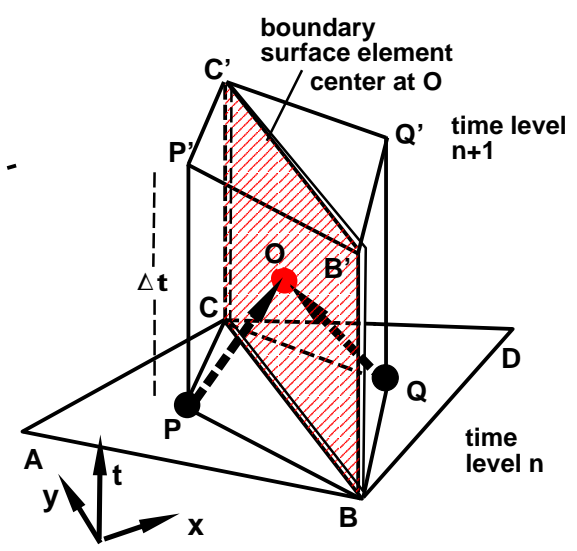

Figure 3: Numerical treatment of NRBC in $E_{3}$.

to $\mathbf{V}_{e}$ in a neighborhood of $O$ at the boundary (in terms of phase and amplitude), and there is no reflection at $O$ across the artificial boundary surface.

The continuity of $\mathbf{V}$, or $\mathbf{U}$ or $\mathbf{W}$ (Eq.(5)- Eq.(7)) across the boundary surface is thus the basic criterion of NRBC adopted in the present paper. In $\S 3.2$, the numerical NRBC (Type I) is constructed based on the continuity criterion. In $\S 4$, the relation between an NRBC and the flux balance across the boundary surface is established. Such relation leads to another absorbing NRBC (Type II).

\section{The numerical treatment of the continuity criterion}

Fig. 3 illustrates a 2-D (in $E_{3}$ ) NRBC treatment. Let $\triangle A B C$ be a boundary cell centered at $P$, with the side $B C$ coincident with the artificial boundary surface. $\triangle B C D$ is the ghost cell centered at $Q$, sharing the boundary edge $B C$ with $\triangle A B C$. Let $O$ be the centroid of the boundary surface element $B C C^{\prime} B^{\prime}$. The limiting process of $\lim _{P_{i} \rightarrow O} \mathbf{U}\left(P_{i}\right)$ is equivalent to extrapolating $\mathbf{U}$ from the interior node $P$ to $O$ by Taylor expansion. Similarly, $\lim _{P_{e} \rightarrow O} \mathbf{U}\left(P_{e}\right)$ is equivalent to extrapolating $\mathbf{U}$ from the exterior ghost node $Q$ to $O$ by Taylor expansion.

Although theoretically, (7) implies up to $C^{\infty}$ continuity, in numerical approximation, only low order continuity such as $C^{0}, C^{1}$ or $C^{2}$, etc. can be achieved. Since a plane wave solution (9) is based on parameters such as its amplitude and phase, the numerical approximation of $\mathbf{V}$ is required to be at least $C^{1}$ continuous at the artificial boundary in order to be consistent with the physical solution. Taking a 1-D version of (9) for example, the $C^{1}$ continuity requirement is explained as follows.

It suffices to consider only a scalar component of $\mathbf{V}$, say, the first one $\rho$. After discretization, the (artificial) boundary surface element center $O$ is used to represent the entire surface element $\Delta s$. Then, from the continuity criterion, approximately, it can be inferred that the 'amplitude' $\left(\tilde{\rho}_{o}\right)_{i}=\left(\tilde{\rho}_{o}\right)_{e}=\tilde{\rho}_{o}$, where the subscripts $o, i$ and $e$ 
stand respectively for the surface center $O$, domain interior and exterior. Approximately, at the boundary surface $\Delta s$,

$$
\rho_{i}=\tilde{\rho}_{o} e^{i\left(k_{i} x_{o}-\omega_{i} t_{o}\right)}=\rho_{e}=\tilde{\rho}_{o} e^{i\left(k_{e} x_{o}-\omega_{e} t_{o}\right)} .
$$

Note that numerically the $C^{0}$ continuity result (10) provides no information about the wave number $k$ and the frequency $\omega$. With the presence of phase error, numerical reflection may still occur. However, if the numerical continuity is enhanced from $C^{0}$ to $C^{1}$, i.e.

$$
\left(\rho_{i}\right)_{x}=i k_{i} \rho_{i}=\left(\rho_{e}\right)_{x}=i k_{e} \rho_{e}, \quad\left(\rho_{i}\right)_{t}=-i \omega_{i} \rho_{i}=\left(\rho_{e}\right)_{t}=-i \omega_{e} \rho_{e}
$$

then $k_{i}=k_{e}$ and $\omega_{i}=\omega_{e}$ and there is no phase error.

In constructing the NRBCs, although any one of $\mathbf{U}, \mathbf{V}$, and $\mathbf{W}$ can be used, $\mathbf{U}$ is selected in the present paper. Therefore, in addition to $\mathbf{U}_{\mathbf{p}}$, the space and time gradients of $\mathbf{U}$ at $P$, namely, $\mathbf{U}_{\mathbf{x}}, \mathbf{U}_{\mathbf{y}}, \mathbf{U}_{\mathbf{z}}$ and $\mathbf{U}_{\mathbf{t}}$ are also required. The resulting linear Taylor expansion ( $C^{1}$ continuity) yields better accuracy and is consistent with the NRBC criterion. The NRBC at the ghost node $Q$ now turns out to be a problem of how to define $\mathbf{U}$ and its gradients at $Q$ so that the flow is $C^{1}$ continuous at the boundary surface (represented by $O$ ).

\subsection{Examples of NRBC - the extrapolation technique}

For the Type I (outflow) NRBC, under a mirror image assumption explained later, it is found that a simple extrapolation technique works well.

First, an example of NRBC in $E_{3}$ (2-D space) for triangular mesh is illustrated. As shown in Fig. 3, assume $\triangle A B C$ is a triangular boundary cell with the edge $B C$ lying on the boundary and conveniently parallel to the $y$-axis. Define a ghost node $D$ as the mirror image of the triangle vertex $A$ with respect to $B C$. Then $\triangle A B C$ and $\triangle D B C$ are mutually mirror images of each other (the mirror image assumption). At time step $n$, conservative variables $\mathbf{U}$ are given at the cell center $P$ of $\triangle A B C$. Then, the NRBC (labeled as Type I) at the geometrical center $Q$ of the ghost cell may be defined as:

$$
(\mathbf{U})_{Q}=(\mathbf{U})_{P},\left(\mathbf{U}_{\mathbf{x}}\right)_{Q}=\left(\mathbf{U}_{\mathbf{x}}\right)_{P}=0,\left(\mathbf{U}_{\mathbf{y}}\right)_{Q}=\left(\mathbf{U}_{\mathbf{y}}\right)_{P}
$$

Apply linear Taylor expansions to domain interior and exterior separately:

$$
\begin{gathered}
\left(\mathbf{U}_{O}\right)_{\text {interior }}=\mathbf{U}_{P}+\left(y_{O}-y_{P}\right)\left(\mathbf{U}_{\mathbf{y}}\right)_{P}+1 / 2 \Delta t\left(\mathbf{U}_{\mathbf{t}}\right)_{P}, \quad\left(\mathbf{U}_{O}\right)_{\text {exterior }}=\mathbf{U}_{Q}+\left(y_{O}-y_{Q}\right)\left(\mathbf{U}_{\mathbf{y}}\right)_{Q}+1 / 2 \Delta t\left(\mathbf{U}_{\mathbf{t}}\right)_{Q} \\
\text { hence, } \quad\left(\mathbf{U}_{O}\right)_{\text {exterior }}=\left(\mathbf{U}_{O}\right)_{\text {interior }} .
\end{gathered}
$$

Here, the subscripts $P, Q$ and $O$ of $x$ and $y$ denote the corresponding coordinates of $P, Q$ and $O$, and from (1), the time partial derivatives $\left(\mathbf{U}_{\mathbf{t}}\right)_{Q}=\left(\mathbf{U}_{\mathbf{t}}\right)_{P}$ can be directly obtained. Thus, $\mathbf{U}$ is $C^{1}$ continuous at $O$ across the boundary surface element, (6) is satisfied and the boundary surface element is non-reflective. 

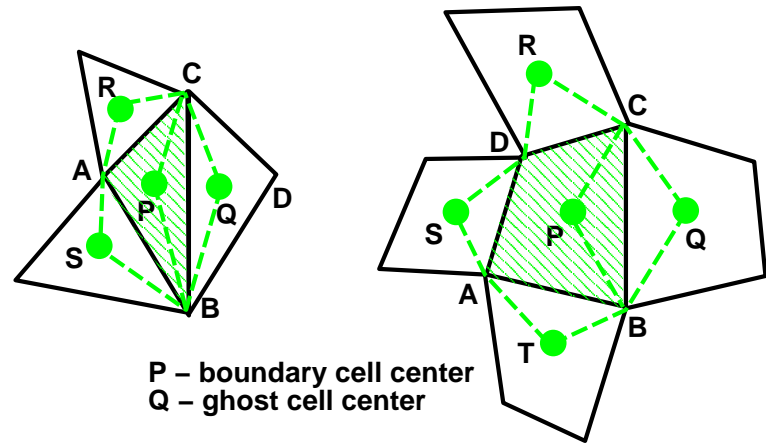

Figure 4: Control volumes $(C V \mathrm{~s})$ in $E_{3}$ for compact updating. Left: boundary cell $\triangle A B C$ and the corresponding hexagon $C V$ base $A S B Q C R A$; right: quadrilateral boundary cell $A B C D$ and its corresponding octagon $C V$ base $A T B Q C R D S A ; R, S, T$ are centers of neighboring cells and $B C$ the boundary. In any case, quadrilateral $P B Q C$ is a portion of the $C V$ base.

In a consistent way, for 3-D flows, under the same mirror image assumption on the ghost cells, the following extrapolations are valid NRBCs with $C^{1}$ continuity:

$$
\mathbf{U}_{\mathbf{Q}}=\mathbf{U}_{\mathbf{p}},\left(\mathbf{U}_{\mathbf{x}}\right)_{\mathbf{Q}}=\left(\mathbf{U}_{\mathbf{x}}\right)_{\mathbf{P}}=\mathbf{0}, \quad\left(\mathbf{U}_{\mathbf{y}}\right)_{\mathbf{Q}}=\left(\mathbf{U}_{\mathbf{y}}\right)_{\mathbf{P}},\left(\mathbf{U}_{\mathbf{z}}\right)_{\mathbf{Q}}=\left(\mathbf{U}_{\mathbf{z}}\right)_{\mathbf{P}}
$$

or

$$
\mathbf{U}_{\mathbf{Q}}=\mathbf{U}_{\mathbf{p}}+\Delta x\left(\mathbf{U}_{\mathbf{x}}\right)_{\mathbf{P}},\left(\mathbf{U}_{\mathbf{x}}\right)_{\mathbf{Q}}=\left(\mathbf{U}_{\mathbf{x}}\right)_{\mathbf{P}}, \quad\left(\mathbf{U}_{\mathbf{y}}\right)_{\mathbf{Q}}=\left(\mathbf{U}_{\mathbf{y}}\right)_{\mathbf{P}},\left(\mathbf{U}_{\mathbf{z}}\right)_{\mathbf{Q}}=\left(\mathbf{U}_{\mathbf{z}}\right)_{\mathbf{P}}
$$

where $\Delta x=x_{Q}-x_{P}$.

As demonstrated in the examples in $\S 6$ and $\S 7$, this Type I NRBC works well for either supersonic or subsonic flows at the outflow boundary, but it should be noted that:

1. (12) or (13) is but a possible selection under the mirror image assumptions, there are other forms of NRBCs based on (5) - (7);

2. The extrapolation technique utilizes the nearby $\mathbf{U}_{P}$ data to approximate the admissible $\mathbf{U}$ data at the artificial boundary, which is not an unreasonable choice, but there is a danger that the solution could drift away from the true solution (see Appendix ) after long time steps marching. A remedy is to incorporate the Type I NRBC with other physical boundary conditions (e.g. back pressure, etc.)

The extrapolation technique is not a new idea, there are many successful examples, such as [9] and [10]. In [10], it was shown in a different way, that the extrapolation NRBC also works well even for subsonic flows.

\subsection{Numerical procedures of NRBC}

The implementation of the NRBC is incorporated in the numerical procedure and may be summarized in the following steps: 


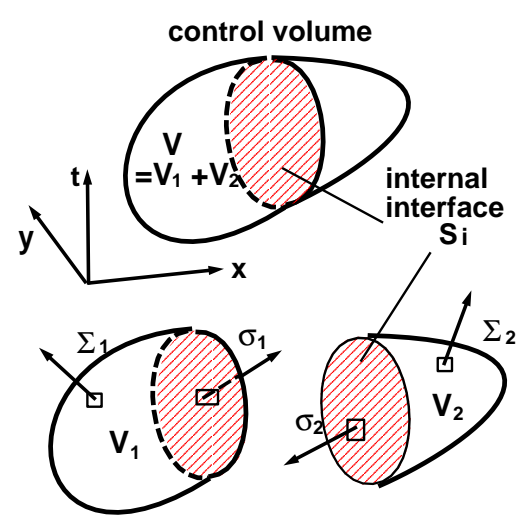

Figure 5: Fluxes balance on an internal face $S_{i}$ in $E_{3}$.

(i) Based on the flow data at the boundary cell center $P$, i.e. $\mathbf{U}_{\mathbf{P}}$ and its spatial gradients (slopes) $\mathbf{U}_{\mathbf{x}}, \mathbf{U}_{\mathbf{y}}$ and $\mathbf{U}_{\mathbf{z}}$, determine the flow data $\mathbf{U}_{\mathbf{Q}}$ as well as its gradients $\mathbf{U}_{\mathbf{x}}, \mathbf{U}_{\mathbf{y}}$ and $\mathbf{U}_{\mathbf{z}}$, i.e. the NRBC at $Q$, as described in $\S 3.1$.

(ii) Update $\mathbf{U}$ at boundary cell center $P$ to the new time level by the conservation laws (2). In order that $\mathbf{U}$ be $C^{1}$ continuous across the artificial boundary, the updating procedure must be carefully designed to take account of the accuracy of surface flux calculation. Here a compact updating procedure described in $\S 3.3$ is recommended.

(iii) After $\mathbf{U}$ at all the interior cell centers of the computational domain are updated, evaluate the new spatial gradients $\mathbf{U}_{\mathbf{x}}, \mathbf{U}_{\mathbf{y}}, \mathbf{U}_{\mathbf{z}}$ at the boundary cell center $P$ by finite difference. Certain flux limiters might be needed in the process. For multi-dimensional flows, a linear equation system is required to solve for the gradients.

(iv) Repeat steps (i) - (iii) and march in time.

\subsection{Compact updating - a viable time marching technique}

The updating procedure recommended here is identical to the one used in the recent CE/SE method (Chang et al $[12,13])$ and similar to the NT (Nessyahu-Tadmor ) scheme [14] in 1-D flow.

The purpose for compact updating is to achieve high accuracy ( $C^{1}$ continuity) from a small cell stencil (e.g. the stencil formed by the immediate neighboring cells). Therefore, not only $\mathbf{U}$ but also its gradients $\mathbf{U}_{x}, \mathbf{U}_{y}, \mathbf{U}_{z}$ and $\mathbf{U}_{t}$ are required. The compact updating is capable of maintaining $C^{1}$ continuity of $\mathbf{U}$ across the artificial boundary.

Consider a triangular cell $\triangle A B C$ in $E_{3}$ (Fig.4, shaded area). Let $P$ and $Q, R, S$ be respectively the cell centers of $\triangle A B C$ and its neighbouring triangular cells. Most of the finite volume schemes use the space-time cylinder $\triangle A B C$ as the control volume $(C V)$ for updating $\mathbf{V}$ or $\mathbf{U}$ at $P$. Surface flux along, say, $A B$ is obtained by extrapolation from $S$ across the cell to the surface center along $A B$. Another treatment is to include $S$ in the $C V$ and replace the flux along $A B$ by the fluxes along $A S$ and $S B$. Since $S$ sits right on both surfaces along $A S$ and $S B$, no extrapolation across the interior of the $C V$ is involved. For the triangular cell $\triangle A B C$ in Fig. 4 the $C V$ turns out to be a space-time hexagon cylinder in $E_{3}$ based on $A S B Q C R A$. By applying the integral conservation laws (2) to the $C V$, updating 
flow data at $P$ based on the flow data at a compact node stencil of $Q, R, S$ is now completed. Fig. 4 also demonstrates that for quadrilateral mesh cells the $C V$ is an octagon cylinder in $E_{3}$ (2-D space).

The surface fluxes for the CV surfaces passing through a vertex, say, cell centers $Q, R, S$, can be evaluated by first extrapolating $\mathbf{U}$ along the surface to their corresponding surface centers by linear Taylor expansion, calculating flux functions $\mathbf{F}, \mathbf{G}$ and $\mathbf{H}$, and then incorporating the surface unit normal vector and computing the fluxes. For high space dimensions, the $C V$ s are geometrically more complicated. More details including the updating of $\mathbf{U}_{x}, \mathbf{U}_{y}$, and $\mathbf{U}_{z}$ can be found in $[12,13]$.

It should be noted that the compact updating is suggested for boundary cells only. For interior cells, one may resume to use other finite volume schemes.

\section{The absorbing NRBC and the matched layer}

Based on the same continuity criterion, another NRBC (Type II) is introduced in this Section. With this NRBC, an interesting extra advantage is, a thin matching layer between the interior flow and the prescribed boundary condition will be formed automatically should any discrepancy is developed between them.

\subsection{Relation between an NRBC and the flux balance across the boundary surface}

In this subsection, the relation between two statements is established. The first one states that the incoming fluxes at the artificial boundary surface are equal to the outgoing fluxes, or fluxes are balanced across the boundary surface. Here, the outgoing flux is defined as a portion of the left hand side of (2):

$$
\int_{\Delta S} \mathbf{I}_{\mathbf{m}} \cdot \mathrm{d} \mathbf{s}, \quad m=1,2,3,4,5,
$$

where $\Delta S$ denotes the artificial boundary surface (element). The second statement is that the boundary is nonreflective. Such a relation leads to the Type II NRBC. Numerical implementation of the Type II NRBC is straightforward and will be illustrated in $\S 4.2$.

Let $V$ be any control volume in the $E_{4}$ space intersected and divided into two portions $V_{1}$ and $V_{2}$ by an internal surface $S_{i}$. Let $\Sigma_{1}, \Sigma_{2}$ and $\sigma_{1}, \sigma_{2}$ be the fluxes around the surfaces of $V_{1}$ and $V_{2}$ respectively. Here $\sigma_{1}$ and $\sigma_{2}$ are the outgoing fluxes at the interface $S_{i}$ for $V_{1}$ and $V_{2}$ respectively (Fig. 5 ). Then, the following lemma holds:

Lemma 1: For a control volume $V$ in the $E_{4}$ space, fluxes passing through any of its internal surface $S_{i}$ are balanced, i.e. $\sigma_{1}+\sigma_{2}=0$.

Proof: Apply (2) to $V, V_{1}$ and $V_{2}$ separately. We have

$$
\Sigma_{1}+\Sigma_{2}=\int_{V} \mathbf{Q}_{\mathbf{m}} \mathrm{d} V, \quad \Sigma_{1}+\sigma_{1}=\int_{V_{1}} \mathbf{Q}_{\mathbf{m}} \mathrm{d} V, \quad \Sigma_{2}+\sigma_{2}=\int_{V_{2}} \mathbf{Q}_{\mathbf{m}} \mathrm{d} V
$$

Add the second and the third equations and then subtract the first one, we have $\sigma_{1}+\sigma_{2}=0$.

In other words, across any interior surfaces, no extra 'source' should be generated. 


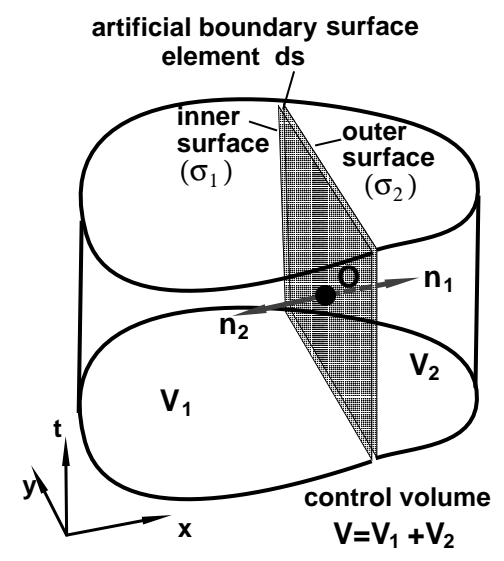

Figure 6: Fluxes balance across a boundary surface element in a control volume (the above figure) in $E_{3}$.

Next, we show that locally the continuity of flow variables (Eq. (5), (6) or (7)) can be inferred from flux balance. Hence, flux balance suffices for an NRBC.

Consider an element $d s$ of the cylindrical spatial boundary surface in $E_{4}$. As shown in the control volume in Fig. 6 (in $E_{3}$ ), ds is centered at $O$. Assume the outgoing unit normal vector at $O$ is $\mathbf{n}_{\mathbf{1}}=\left(n_{x}, n_{y}, n_{z}, 0\right)^{T}$, then, the incoming unit normal is $\mathbf{n}_{\mathbf{2}}=\left(-n_{x},-n_{y},-n_{z}, 0\right)^{T}$. The outgoing flux $\sigma_{\mathbf{1}}$ for the inner boundary surface:

$$
\sigma_{1}=\left(\begin{array}{c}
\sigma_{11} \\
\sigma_{12} \\
\sigma_{13} \\
\sigma_{14} \\
\sigma_{15}
\end{array}\right)=d s\left[n_{x}\left(\begin{array}{c}
F_{1} \\
F_{2} \\
F_{3} \\
F_{4} \\
F_{5}
\end{array}\right)+n_{y}\left(\begin{array}{l}
G_{1} \\
G_{2} \\
G_{3} \\
G_{4} \\
G_{5}
\end{array}\right)+n_{z}\left(\begin{array}{c}
H_{1} \\
H_{2} \\
H_{3} \\
H_{4} \\
H_{5}
\end{array}\right)\right]=d s\left[n_{x} \mathbf{F}+n_{y} \mathbf{G}+n_{z} \mathbf{H}\right] .
$$

Let the outgoing boundary surface flux vector be:

$$
\mathbf{L}=\left[n_{x} \mathbf{F}+n_{y} \mathbf{G}+n_{z} \mathbf{H}\right],
$$

then

$$
\mathbf{L}=\sigma_{1} / d s
$$

Let $\mathbf{V}=(\rho, u, v, w, p)^{T}$ be the primitive flow variable vector. $\mathbf{L}$ may be considered as a non-linear vector function of V. The jacobian matrix $\frac{\partial \mathbf{L}}{\partial \mathbf{V}}$ has the eigen values (e.g. see Hirsch [8], p.177):

$$
\lambda_{1}=\lambda_{2}=\lambda_{3}=u \cdot n_{x}+v \cdot n_{y}+w \cdot n_{z}, \quad \lambda_{4}=\lambda_{1}-c, \quad \lambda_{5}=\lambda_{1}+c .
$$

where $c$ is the speed of sound. If none of the eigenvalues vanishes at $O$, the jacobian $\frac{\partial \mathbf{L}}{\partial \mathbf{V}}$ is non-singular, and an inverse vector function, the primitive flow variables $\mathbf{V}$ as a vector function of the surface flux $\mathbf{L}$ exists. Thus, 


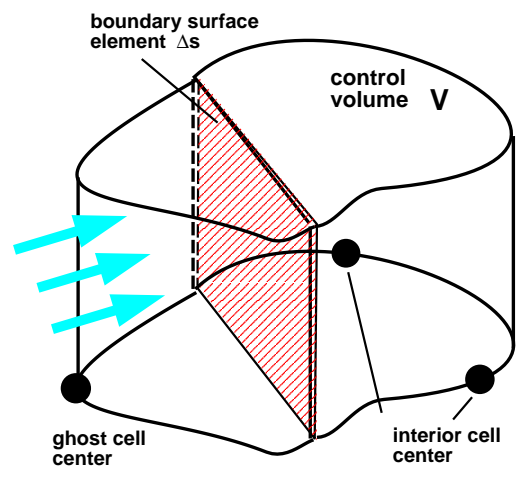

Figure 7: NRBC at inflow in $E_{3}$. Inflow data are given at the ghost cell center.

Lemma 2: If the jacobian $\frac{\partial \mathbf{L}}{\partial \mathbf{V}}$ is non-singular, then, locally, the primitive variables $\mathbf{V}$ are uniquely defined by the flux vector $\mathbf{L}$ at the center $O$ of the boundary surface element $d s$.

Proof: Assume $\mathbf{L}\left(\mathbf{V}_{\mathbf{1}}\right)=\mathbf{L}_{\mathbf{1}}$ and there is another $\mathbf{V}_{\mathbf{2}}$ in the neighborhood of $\mathbf{V}_{\mathbf{1}}$, such that $\mathbf{L}\left(\mathbf{V}_{\mathbf{2}}\right)=\mathbf{L}_{\mathbf{1}}$. By a linear Taylor expansion,

$$
\mathbf{L}\left(\mathbf{V}_{\mathbf{2}}\right)-\mathbf{L}\left(\mathbf{V}_{\mathbf{1}}\right)=\left(\frac{\partial \mathbf{L}}{\partial \mathbf{V}}\right)_{\mathbf{V}=\mathbf{V}_{\mathbf{1}}}\left(\mathbf{V}_{\mathbf{2}}-\mathbf{V}_{\mathbf{1}}\right)+O\left(\left|\mathbf{V}_{\mathbf{2}}-\mathbf{V}_{\mathbf{1}}\right|^{2}\right)=\mathbf{0}
$$

Hence $\mathbf{V}_{\mathbf{2}}-\mathbf{V}_{\mathbf{1}}=\mathbf{0}$ or $\mathbf{V}_{\mathbf{2}}=\mathbf{V}_{\mathbf{1}}$ since the first order term cannot cancel with the second order term.

From Lemma 2 and the NRBC continuity criterion (7), it is inferred that locally, under the condition that the jacobian $\frac{\partial \mathbf{L}}{\partial \mathbf{V}}$ is non-singular, the following lemma holds:

Lemma 3: For hyperbolic conservation laws of gas dynamics, an element of the artificial boundary surface is nonreflective if its outgoing fluxes and incoming fluxes are equal (balanced). Lemma 3 states that conditionally the flux balance across a boundary surface element is a sufficient condition for NRBC.

With the concept of flux balance, there is also an intuitive interpretation of the commonly used terms for NRBC such as 'transparent' and 'absorbing'. If the flux $100 \%$ passes through the boundary surface element (or the flux is balanced), the boundary surface is said to be 'transparent' or 'absorbing' to the fluxes. Chang et al [11] were the first attempting to explain the 1-D NRBC using flux concepts.

\subsection{Implementation of absorbing boundary condition}

The lemmas in $\S 4.1$ can be easily applied to construct the Type II NRBC. In case that at the ghost cell center nodes flow variables must be specified as the given values (e.g. at the inflow boundary, Fig. 7), another type (Type II) of NRBC - absorbing NRBC arises. A control volume $V$ across the boundary surface is needed to apply the divergence theorem (2). As shown in Fig. 7, the ghost cell center lies outside of the domain, and the boundary surface element $\Delta s$ is an internal surface of the control volume $V$, then, from Lemma 1 and Lemma 3, $\Delta s$ is automatically a non-reflective boundary surface element. 
The Type II NRBC states that no extra condition of NRBC is needed with the prescribed flow boundary conditions at the ghost cell center. This Type II NRBC is flexible and valid with practically any cell shapes or configurations. Unlike the Type I extrapolation NRBC, ghost cells are not required to be mirror images of the corresponding boundary cells. However, it is noted that $\mathbf{U}$ is still required to be $C^{1}$ continuous across the artificial boundary surface. Consequently, the compact updating is still recommended for the Type II NRBC.

In many cases, over a long time period, the (subsonic) flow may develop a flow near the inflow boundary that is different from the imposed boundary conditions, or the boundary condition becomes 'overdetermined'. Then, a 'matched layer' may be formed between the computed interior flow and the imposed flow boundary condition (see Fig. 18). The situation is somewhat similar to that in the PML (perfectly matched layer) method $[5,6]$ except that the same set of governing equations are still valid in the layer. The shock-capturing numerical scheme should be able to quickly resolve this non-physical discontinuity in a few cells.

\section{Discussions on the NRBCs}

In practical applications, due to discretization, approximation and lack of information in the domain exterior, there are limitations for both Type I and Type II NRBCs.

For Type I NRBC, as mentioned in $\S 3.1$, extrapolation technique might lead to drifting or deviation from the true solution because no flow data outside the outflow boundary is available. In addition, under the mirror image assumption on the ghost cells in $\S 3.1$ and the assumption that the boundary surface $\Delta s$ is normal to the $x$ axis, the NRBC (12) implies that the NRBC continuity criterion (5 - 7) are satisfied at any point on $\Delta s$. However, as explained in the following, due to discretization and the possible consequent phase error, the accuracy of the NRBC could be degraded.

Consider the Fourier mode in a plane wave solution (9), i.e., $e^{i(\mathbf{k} \bullet \mathbf{x}-\omega t)}$. Here, $\theta(x, t)=\mathbf{k} \bullet \mathbf{x}-\omega t$ is the phase of the wave mode, with $\mathbf{k}$ being the wave number vector in the propagation direction. Generally, the direction of $\mathbf{k}$ may or may not be the same as the flow direction. $\theta(x, t)=$ const. stands for a wavefront ( or a characteristic surface, see e.g. Hirsch [8], p.150, Courant and Hilbert [1] ). After discretization, the center $O$ of $\Delta s$ (Fig. 3) is employed to represent the entire $\Delta s$. Then how much phase error is introduced to the Fourier mode by the discretization? Let $\mathbf{x}=(x, y, z)$ be the position vector of any point on $\Delta s$ and $\mathbf{x}_{O}$ the position vector of the center $O$. For clarity, assume time $t$ is held unchanged. Then, after discretization, the phase error $\Delta \theta$ due to replacing $\mathbf{x}$ by $\mathbf{x}_{O}$ is:

$$
\Delta \theta=\mathbf{k} \bullet \mathbf{x}-\mathbf{k} \bullet \mathbf{x}_{O}=\mathbf{k} \bullet\left(\mathbf{x}-\mathbf{x}_{O}\right)
$$

note that $\Delta \mathrm{x}=\mathrm{x}-\mathbf{x}_{O}$ lies on $\Delta s, \Delta \theta=0$ when $\mathbf{k}$ is normal to $\Delta s$. Therefore, for Type I NRBC, the best result is obtained when the wave propagation direction is normal or only slightly oblique to the boundary surface. Otherwise, a phase error of order $O(\Delta \mathbf{x})$ may be introduced. It deteriorates the accuracy of NRBC and causes numerical reflection. 


\begin{tabular}{ccc}
$\mathrm{x}$ & EXACT & $\mathrm{CE} / \mathrm{SE}$ \\
\hline 430.00 & 0.0000000000 & 0.0000000000 \\
431.00 & 0.0000000000 & 0.0000000000 \\
432.00 & 0.0000000000 & 0.0000000000 \\
433.00 & 0.0000000001 & 0.0000000001 \\
434.00 & 0.0000000014 & 0.0000000014 \\
435.00 & 0.0000000149 & 0.0000000149 \\
436.00 & 0.0000001391 & 0.0000001391 \\
437.00 & 0.0000011125 & 0.0000011125 \\
438.00 & 0.0000076294 & 0.0000076294 \\
439.00 & 0.0000448527 & 0.0000448527 \\
440.00 & 0.0002260436 & 0.0002260436 \\
441.00 & 0.0009765625 & 0.0009765625 \\
442.00 & 0.0036166981 & 0.0036166981 \\
443.00 & 0.0114823007 & 0.0114823007 \\
444.00 & 0.0312500000 & 0.0312500000 \\
445.00 & 0.0729080650 & 0.0729080650 \\
446.00 & 0.1458161299 & 0.1458161299 \\
447.00 & 0.2500000000 & 0.2500000000 \\
448.00 & 0.3674336231 & 0.3674336231 \\
449.00 & 0.4629373561 & 0.4629373561 \\
450.00 & 0.5000000000 & 0.5000000000 \\
451.00 & 0.4629373561 & 0.4629373561
\end{tabular}

Figure 8: Comparison of exact and numerical results at the domain boundary for the 1-D Gaussian pulse problem.

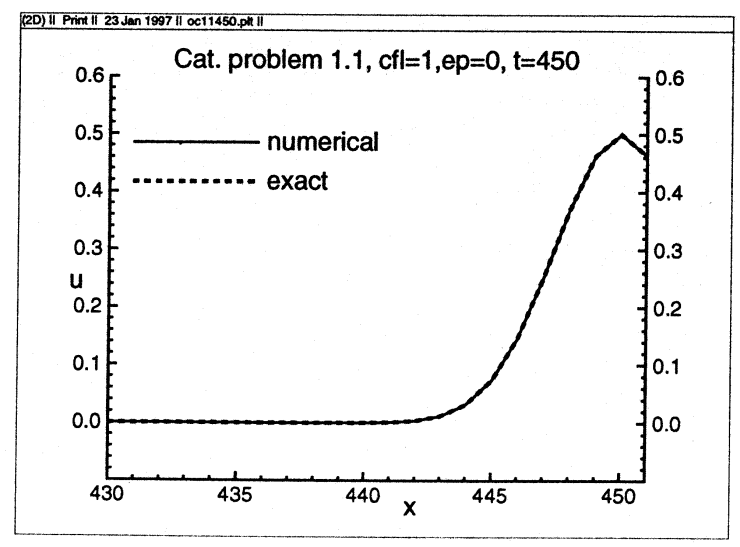

Figure 9: Propagation of 1-D Gaussian pulse at the domain boundary.

For Type II NRBC, in addition to the similar phase error of Type I NRBC, there are other restrictions too. In $\S 4.1$, Lemma 3 is conditionally valid because it is based on the one to one correspondence between the boundary surface flux vector $\mathbf{L}$ and the primitive flow variable vector $\mathbf{V}$. The latter relies on the non-singularity of the jacobian $\frac{\partial \mathbf{L}}{\partial \mathbf{V}}$. In case that $\frac{\partial \mathbf{L}}{\partial \mathbf{V}}$ is singular, Lemma 3 may fail. In addition, Lemma 3 is valid only locally. Globally, the relation between $\mathbf{L}$ and $\mathbf{V}$ involves a quadratic equation, the vector function $\mathbf{V}(\mathbf{L})$ could be multi-valued. This could break the one to one correspondence between $\mathbf{L}$ and $\mathbf{V}$ globally and lead to the failure of Lemma 3 in the global sense.

In a nutshell, in reality, there are various situations that the Type I or II NRBCs can only be partially or approximately implemented, causing spurious reflections at the (artificial) boundary or deviation from the true solution. In practice, an effective remedy is to impose a buffer/sponge zone between the boundary and the interior domain. Although the same governing equations (1) or (2) are employed in the sponge zone, numerical damping is highly increased to 
diminish the wave/disturbance amplitude before it reaches the boundary and to minimize the spurious reflection. An example is depicted in $\S 8$.

\section{Numerical examples for outflow NRBC}

In this Section and $\S 7$, the effectiveness of these NRBCs is demonstrated in numerical examples in one, two and three dimensional spaces.

In principle, any finite volume scheme can be used with the above NRBC if it can be manipulated at certain high accuracy. Here the recently developed space-time conservation element and solution element (CE/SE) method [12,13] is chosen for computing the examples since the compact updating is a standard procedure in the scheme, making application of the NRBCs straightforward and effective. Full details of the method are described in [12,13]. The Type I NRBCs used with the CE/SE method are identical to (12), with possible minor modification according to the grid layout.

\subsection{Propagation of a 1-D Gaussian pulse}

Consider a scalar initial value problem:

$$
\frac{\partial u}{\partial t}+\frac{\partial u}{\partial x}=0
$$

over the range $-20 \leqslant x \leqslant 450$, with a Gaussian pulse $u=0.5 \exp \left[-(\ln 2)\left(\frac{x}{3}\right)^{2}\right]$ at $t=0$. This is one of the benchmark problems of the 1st CAA Workshop [15]( Category 1, Problem 1). The exact solution given there is:

$$
u=0.5 \exp \left[-(\ln 2)\left(\frac{x-t}{3}\right)^{2}\right]
$$

In this example, $\Delta x=1$ is chosen and $\Delta t=1$ is based on $C F L$ number $=1$. With $C F L$ number $=1$, and other parameters $\epsilon=0$ and $\alpha=0$, the CE/SE scheme yields a numerical result which is identical to the exact solution in the interior of the domain $-20 \leqslant x \leqslant 451$. Thus, performance of the outflow Type I NRBC at $x=451$ can be easily validated. At $t=450$, the Gaussian pulse is passing through the outflow boundary $x=451$, where the Type I NRBC (12) with appropriate modification to 1-D flow is imposed. The table in Fig. 8 lists the exact solution and the CE/SE result from $x=430$ to $x=451$. The result is also plotted in Fig. 9. It is seen that they are completely identical (up to 10 decimal places) and there is absolutely no reflection, although the grid is rather crude with $\Delta x=1$.

\subsection{2-D free shear layer instability and vortex roll-up}

The problem considered here is identical to the inviscid free shear layer instability problem considered in [16] (Fig. 10). The background mean flow consists of a fast stream (supersonic) in the upper half domain and a slow stream (subsonic) in the lower half domain. The two parallel streams are connected by a continuously changing shear layer of the hyperbolic tangent profile. 


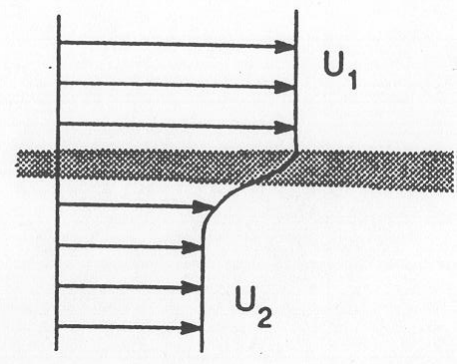

Figure 10: Free shear layer instability problem, $u_{1}=U_{1}=1, v_{1}=0, p_{1}=1 / 3.15, \rho_{1}=1, M_{1}=1.5, u_{2}=$ $U_{2}=.7391304, v_{2}=0, p_{2}=1 / 3.15, \rho_{2}=0.5405405$, with subscripts 1,2 denoting the fast and slow streams respectively.

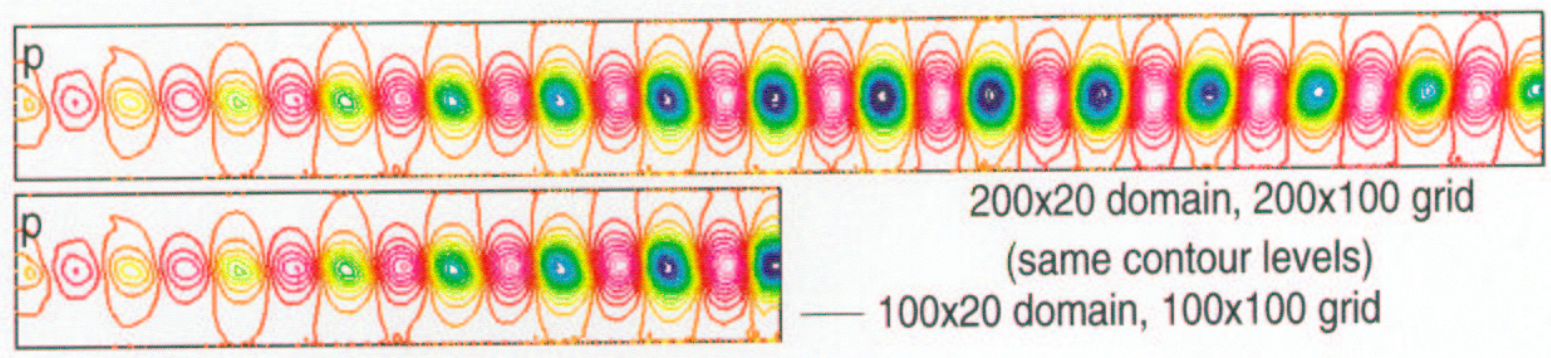

comparison of numerical results with different computational domains, showing effectiveness of outflow NRBC
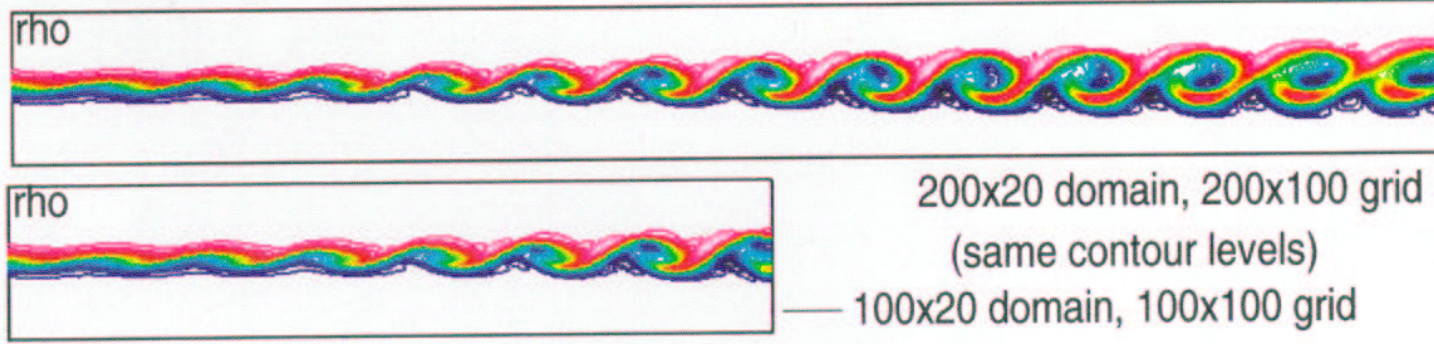

Figure 11: Contours for long and short domains, showing effectiveness of the outflow NRBC. 

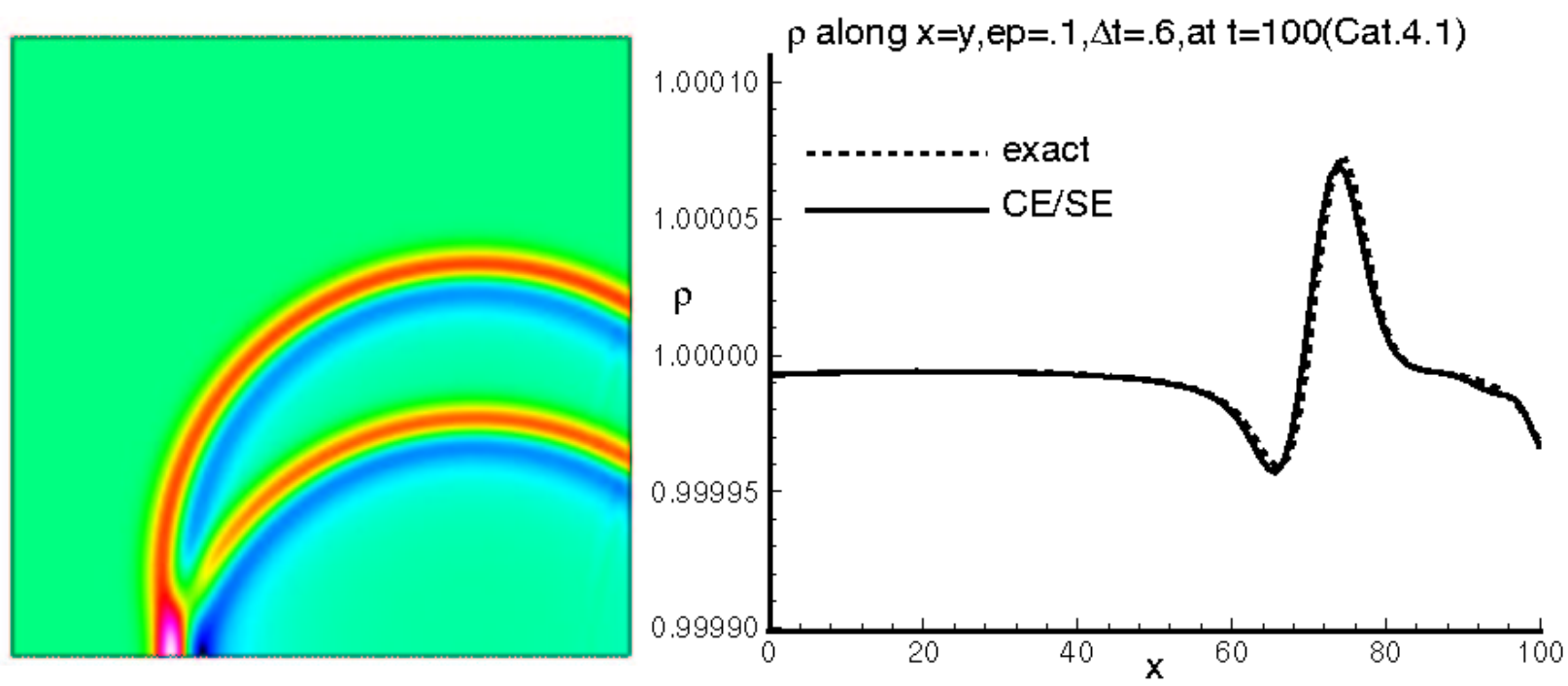

Figure 12: An acoustic pulse above a solid surface passing through the outflow boundary.

In the test, two computational domains are chosen. The first one is $0 \leqslant x \leqslant 200$ and $-10 \leqslant y \leqslant 10$, with a grid of $200 \times 100$ uniform cells. The second one is $0 \leqslant x \leqslant 100$ and $-10 \leqslant y \leqslant 10$, with a grid of $100 \times 100$ uniform cells. Both cases have exactly the same grid cell sizes, time step size $\Delta t=0.15$, and parameters $\epsilon=0.2, \alpha=0$. They are both run for 4000 time steps when the spatial instability is fully developed. To ensure that the instability waves and vortex roll-up develop quickly, a large perturbation amplitude of 0.02 at the most unstable frequency is chosen for the eigenfunctions. At the outflow boundaries, the Type I outflow NRBC is used. Figure 11 demonstrates snapshots of the isobars and isopycnics in the two cases. These contours are observed to be almost identical to each other in their common domain portion. The contours in the short domain seem as if they were a piece chopped off from the longer one. This shows that the outflow NRBC in this case is nearly perfect.

\subsection{Acoustic Pulse Propagation}

This problem is a typical subsonic wave propagation problem [15]. The computational domain in the $x-y$ plane is a square with $-100 \leqslant x \leqslant 100$, and $0 \leqslant y \leqslant 200$. A uniform $201 \times 201$ (triangulated) grid is used with $\Delta x=\Delta y=1$ . Initially, a Gaussian acoustic pulse is located at the lower portion of the domain $(x=0, y=25)$, with a mean flow of Mach number $M=0.5$ in $x$ direction and a solid wall at the bottom. At the other three boundaries, Type II NRBC is used. By choosing a small amplitude factor $\delta=0.001$, the Euler equations are practically linearized. Fig. 12 shows the isobars at $t=100$ and the comparison between numerical and analytical results for density along the line $x=y$ for $0 \leqslant x \leqslant 100$. Although the wave propagation direction is oblique to the outflow boundary, only tiny reflection is observed from Fig. 12. Another 2-D example with completely subsonic flow will be illustrated in $\S 7$. 


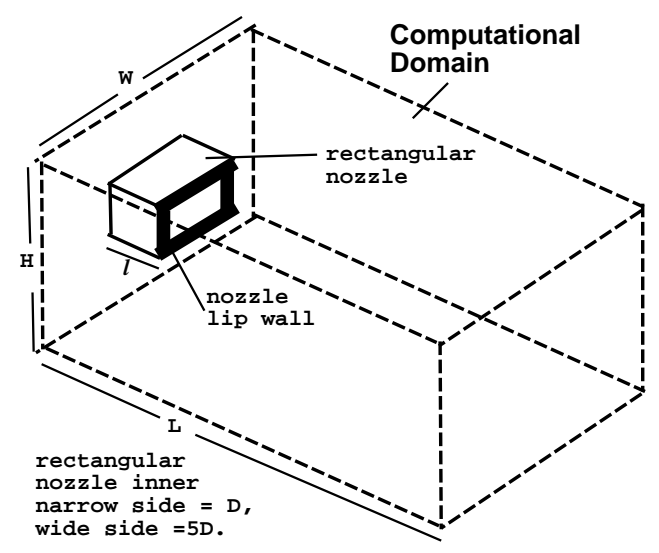

Figure 13: Sketch of the rectangular jet, aspect ratio 5, jet Mach number $M_{j}=1.6, L=16 D, W=16 D$ and $H=5.6 D$.

\subsection{3-D rectangular jet flow}

Fig. 13 is the sketch of an underexpanded rectangular jet in 3-D space. The rectangular nozzle protrudes into the computational domain by $l=2 D, D$ being the width of the jet. The unstructured mesh consists of about 1.7 million tetrahedral cells. At the inlet plane, ambient (stationary) condition is specified. Jet flow at higher pressure is specified at the nozzle exit. All the rest boundaries are either Type I or Type II NRBC. Fig.s 14 shows snapshots of the isobars and $v$ velocity contours on the cross sectional mid-planes after running 60,000 time steps. Fig. 15 demonstrates the 3-D pressure iso-surfaces. No visible reflection is observed.

\subsection{Influence of the NRBC to the numerical accuracy}

In $\S 3.1$ and $\S 5$, it is commented that since the admissible $\mathbf{V}$ at the artificial boundary is not available and has to be approximated by extrapolation, this will eventually lead to deviation or drifting of $\mathbf{V}$ from the true solution. But in realty, in many cases, the influence from the boundary to the interior flow is small. An example is shown here.

Fig. 16 demonstrates the sound intensity level computed based on two domains with different lengths but same width for the 2-D Mach radiation problem in the 3rd CAA Workshop (Category 5) [18]. The short domain has a grid of $289 \times 144$ nodes. The only difference is that the longer domain has 30 more uniformly distributed nodes added in the $x$ direction downstream. Fig. 16 shows the sound intensity levels ( square of r.m.s. $p^{\prime}$ - pressure fluctuation ) along the line $y=10$ at $t=400$ (40000 time steps or 28 periods ) for both domains. It is observed that the maximum difference is about $2 \times 10^{-10}$, far below the discretization error, thus is negligible. This case also demonstrates the relative drifting side effect of the extrapolation Type I NRBC, but the error is acceptable. 

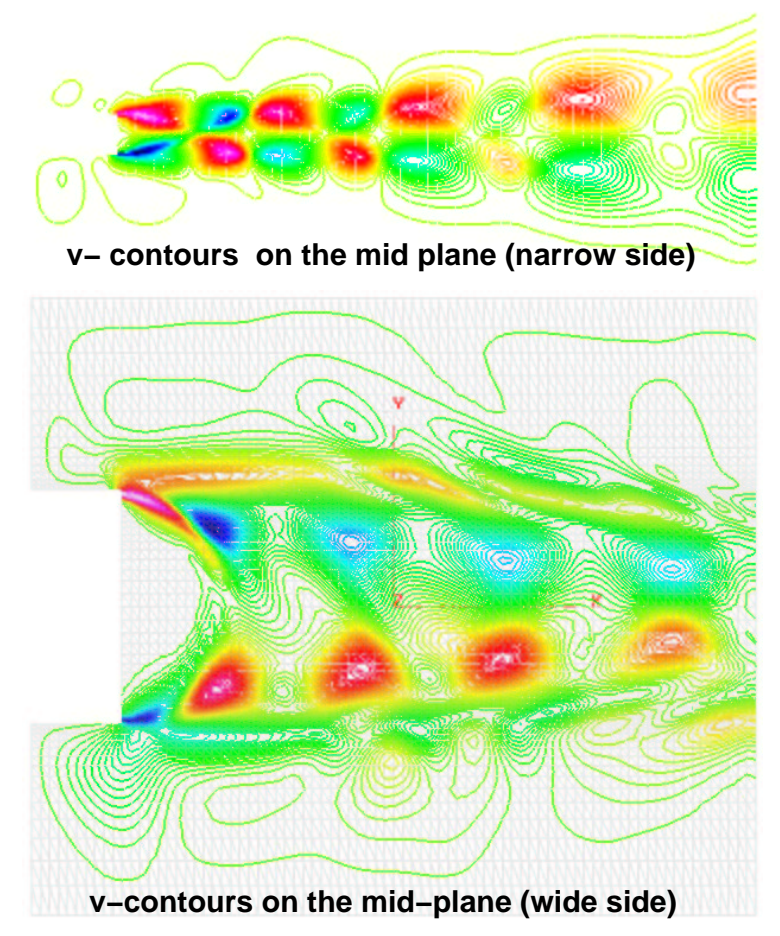

Figure 14: $v$ velocity contours on the mid-planes with mesh background. No visible reflection is observed. At the outflow boundary, flow is supersonic in the jet core and then becomes subsonic across the thick shear layer.

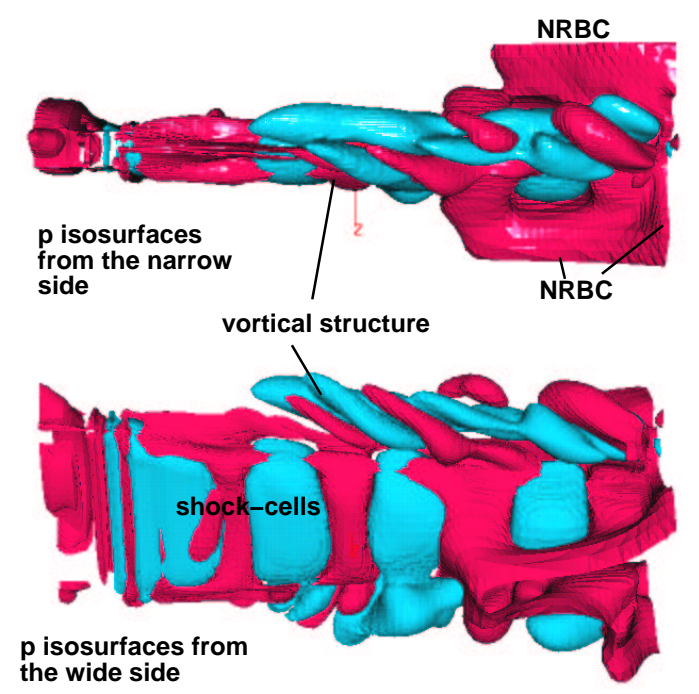

Figure 15: Pressure iso-surfaces, no reflection is observed. 


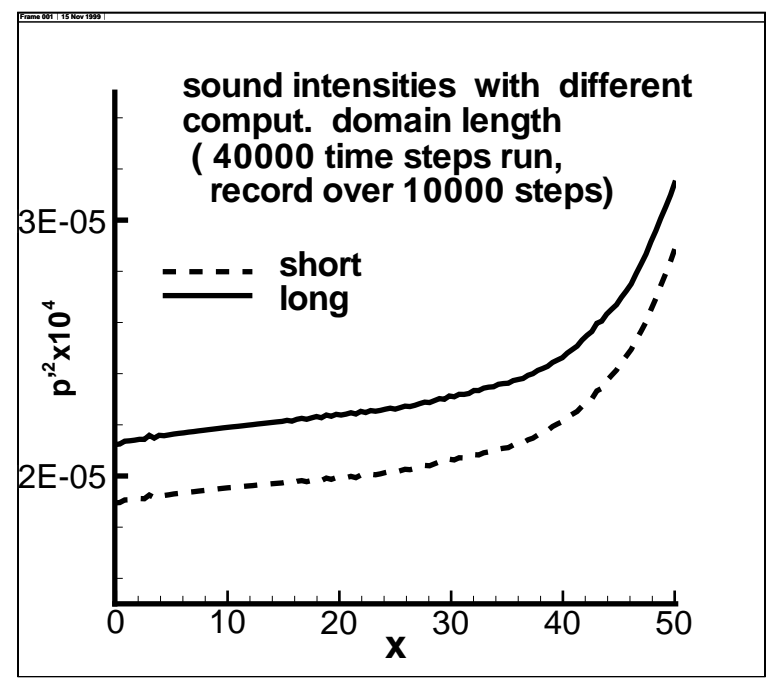

Figure 16: Comparison of sound intensity levels from computational domains of different lengths, showing that the outflow NRBC has negligible influence to the interior.

\section{Numerical example for the absorbing boundary condition}

As stated in $\S 4.2$, for an absorbing boundary condition such as the inlet, the prescribed inlet conditions are already good enough for an NRBC. Fig. 17 illustrates the instantaneous isobars for a cavity noise problem. 42, 000 triangulated structured cells are used. The problem is a $\mathrm{M}=0.8$ flow past a rectangular cavity of aspect ratio of 6.5 . At the cavity walls, no slip boundary condition is imposed. Due to vortex shedding and acoustic feedback at the cavity edges, strong nonlinear acoustic waves are generated and propagate in both upstream and downstream directions [17]. Fig. 18 is an enlargement of Fig. 17 around the inlet area. The details of the contours at the matched layer is revealed. It is observed that there is no spurious reflection and that the matched layer is about 4-5 cells' thick. The matched layer in the Type II NRBC is somewhat similar to that of the PML (perfectly matched layer) method $[5,6]$ in that the difference diminishes quickly within this layer. But the layer arises automatically and there is no need to solve a new set of equations in the layer or to impose any conditions other than the prescribed inflow physical conditions.

For NRBC at the top of the computational domain, a Type I NRBC may be modified from (12) by simply exchanging the axes $x$ and $y: \mathbf{U}_{\mathbf{Q}}=\mathbf{U}_{\mathbf{p}},\left(\mathbf{U}_{\mathbf{y}}\right)_{\mathbf{Q}}=\left(\mathbf{U}_{\mathbf{x}}\right)_{\mathbf{Q}}=\mathbf{0}$, where as before, $P$ and $Q$ are respectively the boundary cell center and the ghost cell center at the top boundary. From Fig.s 17-18, even when the acoustic wave is oblique to the boundary, there is still no visible reflection. A Type I NRBC is applied to the outflow boundary, still, no visible reflection is found for the subsonic flow. 


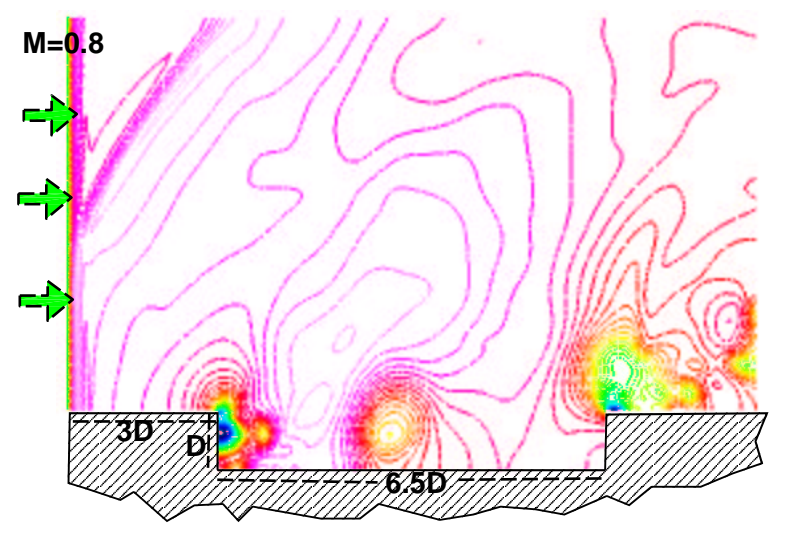

Figure 17: Isobars snapshot for a cavity noise problem (Mach number $M=0.8$ ), showing inflow NRBC and its absorbing property. No visible reflection is found at the top and the outflow boundary
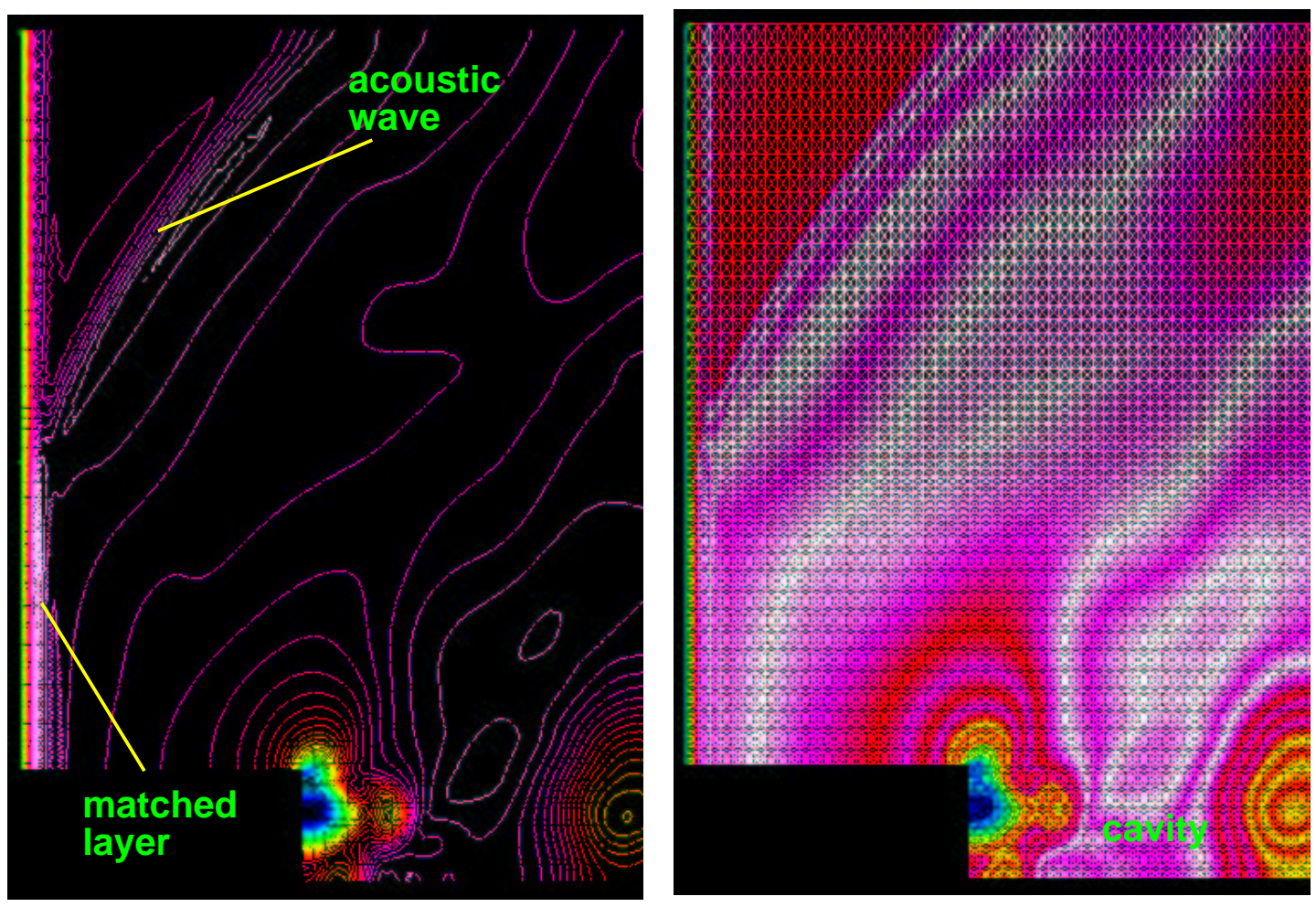

Figure 18: Details of the contours at the inflow boundary, showing the matched layer at the inlet and its spreading over the grid. 


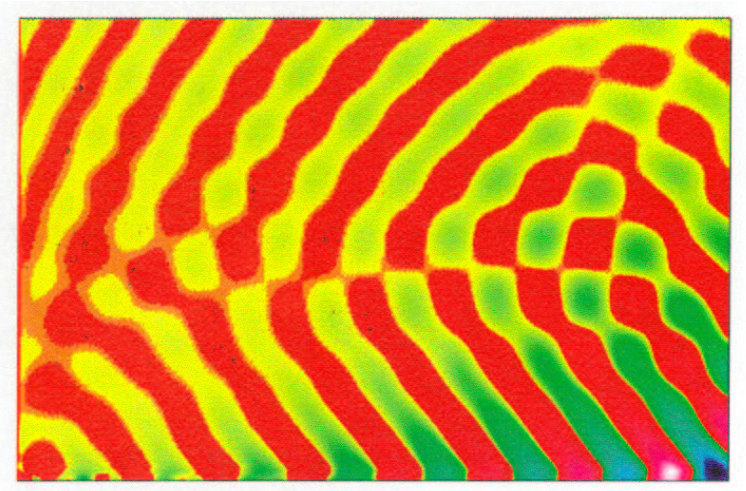

Figure 19: Mach radiation from a $M=2$ axisymmetric jet (without buffer zone), showing severe spurious numerical reflection.

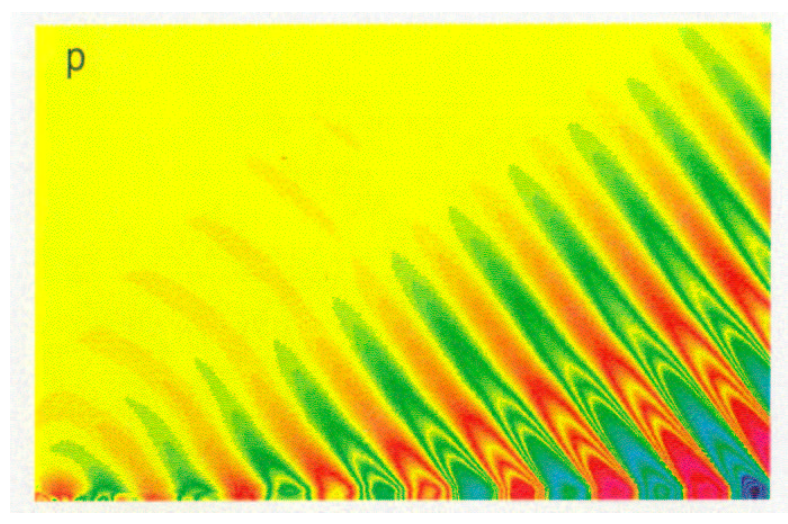

Figure 20: Mach radiation from a $M=2$ axisymmetric jet (with buffer zone but not shown), showing a clean acoustic field.

\section{Application of the buffer/sponge zone}

Generally, the NRBCs amount to little reflection. However, there are situations that they may fail and discernible reflections occur, such as the cases discussed in $\S 5$. A simple but effective remedy is to add a buffer/sponge zone between the core domain and the boundary. In the buffer zone, the same governing equations are still used, except that numerical damping is highly increased. Here, the cell size in the buffer zone may grow rapidly (e.g. exponentially ) and create larger numerical damping. Typically, the number of cells in a buffer zone may vary from a few to 20 . An example of a 2-D axisymmetric jet with Mach radiation from an externally stimulated shear layer is demonstrated. It is similar to the one described in the 3rd CAA workshop benchmark problems (Category 5) [18]. The domain size is $33 D \times 19 D$ with $D$ being the jet nozzle diameter. $300 \times 280$ non-uniform rectangular cells are used before they are further triangulated. Initially, a Mach number $M=2$ jet exists. At the center of the nozzle exit plane, a source is imposed and perturbs the jet flow with a small amplitude $A=0.001$ at a Strouhal number $S t=0.2$. Mach radiation 
is then triggered and gains its strength along the stream. At the outflow boundary, particularly at the shear layer, due to the staggered type mesh and the strong Mach waves with oblique angles, the Type I NRBC fails and spurious reflection is generated and propagates upstream (Fig. 19). After a buffer zone of 10 cells is added at the domain outflow boundary, the spurious reflection disappears and a clean mach wave radiation is shown (Fig. 20). The size of the buffer zone cells grows exponentially at a rate of $20 \%$ along the $x$ axis.

\section{Concluding Remarks}

In the present paper, based on the first principle of non-reflecting, the propagation of plane waves and the hyperbolicity of the Euler equation system are revisited, and then combined to derive the continuity criterion of NRBC. This continuity criterion reveals the nature of NRBC and it is possible to apply it to hyperbolic systems other than the Euler equations. Numerically, $C^{1}$ continuity is required at the artificial boundary to be consistent with the criterion and achieve non-reflection.

Two basic types of NRBCs are described: the extrapolation (Type I) and the absorbing (Type II) NRBCs. Simple but effective $C^{1}$ continuity NRBCs are consequently developed. Generally, their performances are similar to those of the characteristics-based NRBCs. For shock-capturing schemes, Type II NRBC often forms a matched layer within a stencil of a few cells, should discrepancy develops between the interior flow and the boundary conditions. This matched layer is similar to the well-known PML (perfectly matched layer) in [5,6]. Limitations of the NRBCs are also discussed. In particular, the Type I extrapolation NRBCs may cause solution drifting due to lack of information beyond the outflow boundary (see also [10]). A remedy is to incorporate the physical boundary conditions or to use a sponge zone (cf. Appendix).

The diversity of various NRBCs in flow computations can never be overestimated. The purpose of the present paper is to show some guidelines in developing NRBCs that are simple and robust for practical computations. The compact updating procedure proves to work well with the NRBCs and provides $C^{1}$ accuracy in surface flux evaluation. But it is definitely not the only way to achieve non-reflecting effect. Different schemes may have different treatments, see for example, $[9,10]$. Sometimes, a combination of the NRBC treatments may provide much improved results, such as the incorporation of the buffer zone. As a byproduct, we are now also able to explain why the NRBCs with the recently developed CE/SE scheme $[12,13,16,17]$ are robust.

The restriction set in $\S 2$ that the flow is continuous may be lifted, since on the one hand, for shock-capturing schemes, a discontinuity may be considered as a continuous wave with steeper gradient, and on the other hand, a sharp gradient may be decomposed by Fourier series or Fourier integral. But if the wave propagation direction is oblique to the (artificial) boundary, the NRBC may perform poorly due to reasons explained in $\S 5$. Chang et al [11] presented a 1-D example ( i.e. wave propagation direction normal to the boundary) showing how a shock passes through an artificial boundary without causing visible reflection. 


\section{References}

[1] Courant, R. and Hilbert,D. “ Methods of Mathematical Physics”, Vol.II, John Wiley \& Sons, London, 1962.

[2] Givoli,D., “Non-reflecting Boundary Conditions”, J. Comput. Phys., Vol. 94, 1-29(1991).

[3] Engquist, B. and Majda, A., "Absorbing boundary conditions for the numerical simulation of waves", Math Comp., Vol. 31, pp. 629-651 (1977).

[4] Thompson, K. W. "Time-dependent boundary conditions for hyperbolic systems", i \& ii, J. Comput. Phys., Vol. 68, pp. 1-24, (1987); also Vol 89, pp 439-461, (1990).

[5] Hu, F.Q., “On Absorbing Boundary Conditions for Linearized Euler Equations by a Perfectly Matched Layer”, J. Comput. Phys., Vol.129, 201-219 (1996).

[6] Hesthaven, J.S., “The Analysis and Construction of Perfectly Matched Layers for the Linearized Euler Equations”, ICASE Report No. 97-49 (1997) (also in J. Comput. Phys.)

[7] Hedstrom,G.W., “ Nonreflecting Boundary Conditions for Nonlinear Hyperbolic Systems”, J. Comput. Phys. , Vol.30, 222-237 (1979).

[8] Hirsch, C. "Numerical Computation of Internal and External Flows", Vol. I and II, John Wiley \& Sons (1993).

[9] Leveque, R. J., "Finite Volume Methods for Hyperbolic Problems”, pp. 488-489, Cambridge University Press, 2002.

[10] Nordstrom, J., "Accurate Solutions of the Navier-Stokes Equations Despite Unknown Outflow Boundary Data", J. Comput. Phys., Vol.120, 184-205 (1995).

[11] Chang, S. C., Himansu, A., Loh, C. Y., Wang, X. Y., Yu, S.-T. and Jorgenson, P. C. E. "Robust and Simple Non-Reflecting Boundary Conditions for the Space-Time Conservation Element and Solution Element Method ", AIAA Paper 97-2077 (1997).

[12] Chang, S. C., Wang, X. Y. and Chow, C. Y., “ The Space-Time Conservation Element and Solution Element Method - A New High-Resolution and Genuine Multidimensional Paradigm for Solving Conservation Laws ”, $J$. Comput. Phys. vol. 156, pp. 89-136 (1999).

[13] Wang, X.-Y. and Chang S.-C., “ A 2-D Non-splitting Unstructured Triangular Mesh Euler Solver Based on the Space-Time Conservation Element and Solution Element Method" CFD J. vol. 8, pp309-325 (1999).

[14] Nessyahu, H. and Tadmor, E. "Non-oscillatory Central Diffewrencing for Hyperbolic Conservation Laws", $J$. Comput. Phys. , Vol.87, 408-463 (1990). 
[15] "ICASE/LaRC Workshop on Benchmark Problems in Computational Aeroacoustics", Eds. J.Hardin, J.R.Ristorcelli, and C.K.W.Tam, NASA/CP-3300 (1994).

[16] Loh, C. Y., Hultgren, L. S. and Chang S.-C., "Computing Waves in Compressible Flow Using the Space-Time Conservation Element Solution Element Method,” AIAA J., Vol. 39, pp. 794-801 (2001).

[17] Loh, Ching Y., Wang, Xiao Y., Chang, Sin-Chung and Jorgenson, Philip C.E., "Computation of Feedback Aeroacoustic System by the CE/SE Method", in proceedings of the 1st Int'1 Conf. on CFD (ICCFD), Kyoto, Japan, July, 2000; (published by Springer-Verlag, 2001).

[18] “Third Computational Aeroacoustics Workshop on Benchmark Problems”, NASA/CP-2000-209790.

\section{Appendix: Discussions on the continuity criterion and the extrapolation technique}

Without loss of generality, consider a 1-D flow shown in Fig. 21. As a pure initial value Cauchy problem, $\mathbf{V}=$ $\mathbf{V}(x, 0)$ at $t=0$ is given. This problem is well-posed and there exists a unique solution $\mathbf{V}=\mathbf{V}$ o $(x, t)$ over the entire domain $D:-\infty<x<\infty, \quad t \geqslant 0$. If a boundary exists on the left inlet side, the problem becomes an initial-boundary-value problem, a boundary condition is required at the inlet boundary.

Assume the artificial boundary locates at $x=0$ and bisects the entire domain $D$ into domain interior $D_{i}$ and domain exterior $D_{e}: D=D_{i}+D_{e}$ (see $\left.\S 2.4\right)$. In order that $\mathbf{V}_{i}$ and $\mathbf{V}_{e}$ in $\S 2.4$ are respectively the unique well-posed solutions in subdomains $D_{i}$ and $D_{e}$, an admissible common boundary condition they share at $x=0$ is:

$$
\mathbf{V}_{i}(0, t)=\mathbf{V}_{e}(0, t)=\mathbf{V}_{o}(0, t)
$$

Here, $\mathbf{V}_{o}(0, t)$ is the entire domain solution along the line $x=0$; or equivalently, $\mathbf{V}_{o}(0, t)$ may be obtained by the method of characteristics as sketched in Fig. 21 at point $O$ at the boundary.

However, for NRBC problems in reality, $\mathbf{V}_{o}(x, t), \quad x \geqslant 0$; or $\mathbf{V}_{e}(x, t)$ is totally missing (otherwise there is no need to investigate the NRBCs). In this situation, the extrapolation technique e.g. (12) is not an unreasonable choice

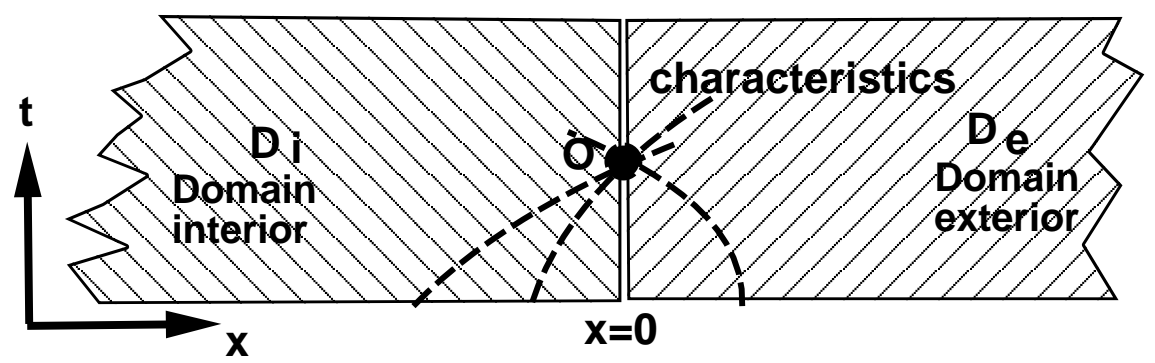

Figure 21: Continuity criterion in 1-D flow. 
for the NRBC continuity criterion. The initial-boundary value problem for $\mathbf{V}_{i}$ is well-posed only when the flow is supersonic or is known to remain unchanged across the artificial boundary at $x=0$ by a priori information. Otherwise, even though locally the extrapolation (12) leads to non-reflecting effect, globally the 'solution' will keep drifting away and deviate from the true solution. The following are some remedies for practical numerical flow computations:

[1] use a sponge (buffer) zone with highly increased numerical damping to filter away the wave ingredients in the flow; when the Type I extrapolation NRBC is applied to the outer outflow boundary, the flow is already uniform at the boundary.

[2] incorporate the extrapolation with other physical boundary conditions (e.g. back pressure etc.).

Notice that the present discussions do not apply to the Type II NRBCs. 


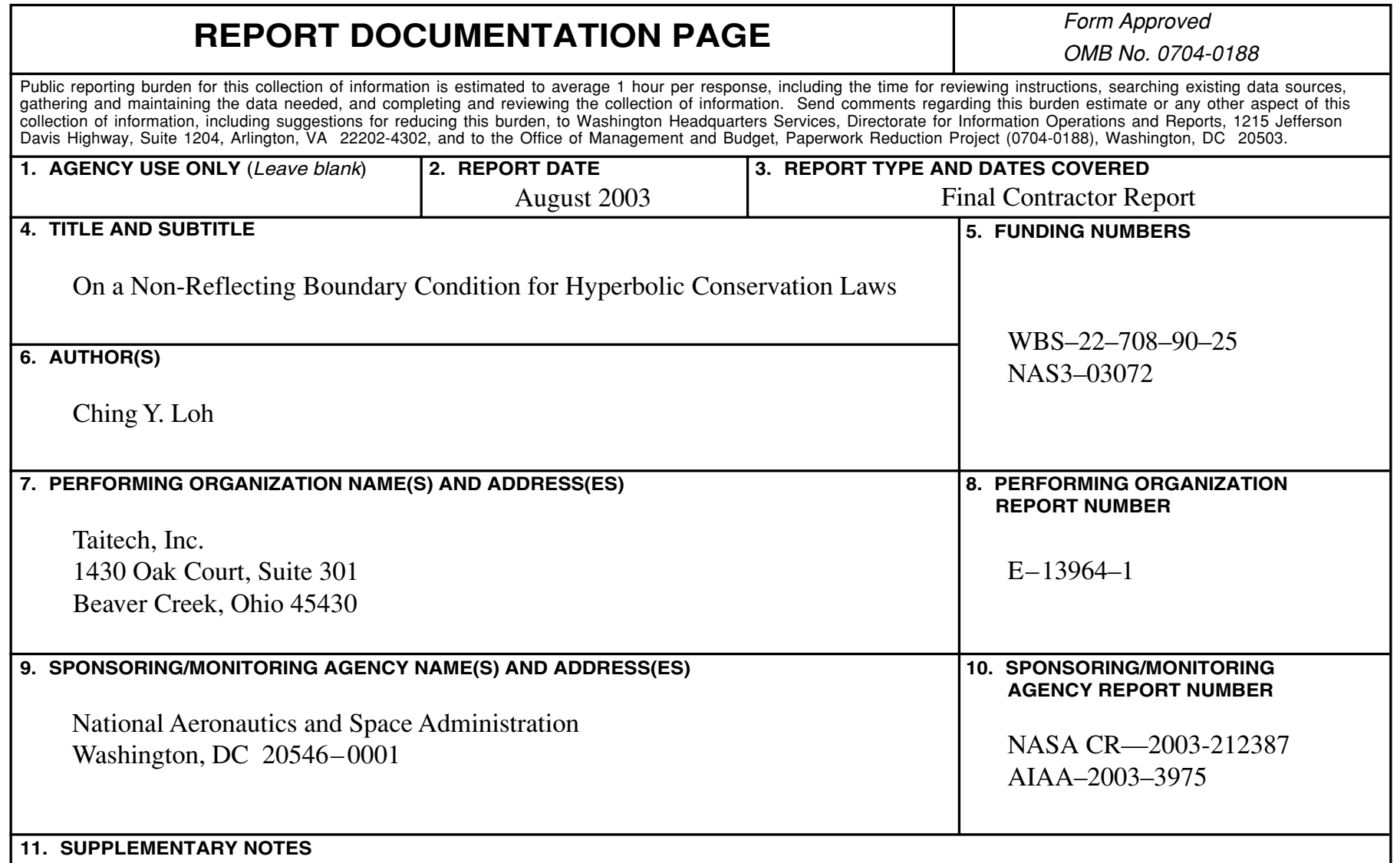

Prepared for the 16th Computational Fluid Dynamics Conference and the 33rd Fluid Dynamics Conference and Exhibit sponsored by the American Institute of Aeronautics and Astronautics, Orlando, Florida, June 23-26, 2003. Project Manager, Richard A. Blech, Turbomachinery and Propulsion Systems Division, NASA Glenn Research Center, organization code 5880, 216-433-3657.

12a. DISTRIBUTION/AVAILABILITY STATEMENT

12b. DISTRIBUTION CODE

Unclassified - Unlimited

Subject Categories: 01, 64, and 67

Distribution: Nonstandard

Available electronically at http://gltrs.grc.nasa.gov

This publication is available from the NASA Center for AeroSpace Information, 301-621-0390.

13. ABSTRACT (Maximum 200 words)

A non-reflecting boundary condition (NRBC) for practical computations in fluid dynamics and aeroacoustics is presented. The technique is based on the first principle of non-reflecting, plane wave propagation and the hyperbolicity of the Euler equation system. The NRBC is simple and effective, provided the numerical scheme maintains locally a $C^{1}$ continuous solution at the boundary. Several numerical examples in 1D, 2D, and 3D space are illustrated to demonstrate its robustness in practical computations.

\section{SUBJECT TERMS}

Nonreflecting boundary condition; Continuity criterion; Hyperbolic conservation laws

\begin{tabular}{|c|c|c|c|}
\hline $\begin{array}{c}\text { 17. SECURITY CLASSIFICATION } \\
\text { OF REPORT } \\
\text { Unclassified }\end{array}$ & $\begin{array}{c}\text { 18. SECURITY CLASSIFICATION } \\
\text { OF THIS PAGE } \\
\text { Unclassified }\end{array}$ & $\begin{array}{c}\text { 19. SECURITY CLASSIFICATION } \\
\text { OF ABSTRACT } \\
\text { Unclassified }\end{array}$ \\
\hline
\end{tabular}

NSN 7540-01-280-5500 